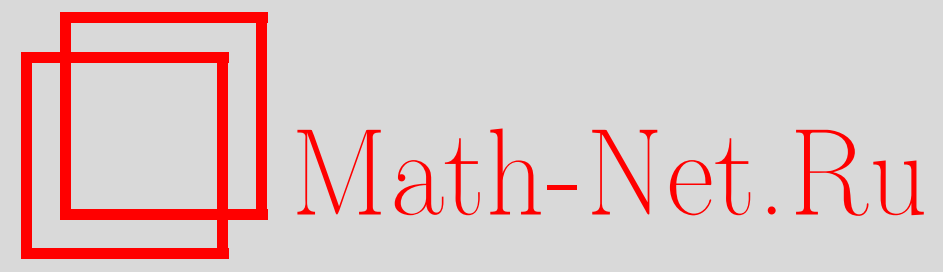

K. Glau, Classification of Lévy processes with parabolic Kolmogorov backward equations, Теория вероятн. и ее примен., 2015, том 60, выпуск 3, 525-552

DOI: https://doi.org/10.4213/tvp4636

Использование Общероссийского математического портала Math-Net.Ru подразумевает, что вы прочитали и согласны с пользовательским соглашением http://www . mathnet.ru/rus/agreement

Параметры загрузки:

IP: 18.234 .197 .8

26 апреля 2023 г., 15:54:08

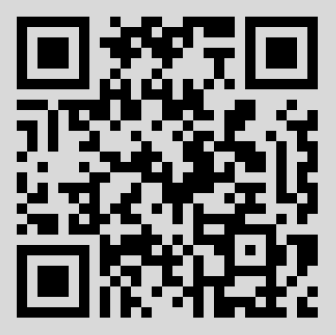




\title{
CLASSIFICATION OF LÉVY PROCESSES WITH PARABOLIC KOLMOGOROV BACKWARD EQUATIONS ${ }^{1)}$
}

\begin{abstract}
Процессы Леви классифицируются в соответствии с пространствами решений ассоциированных интегро-дифференциальных уравнений с частными производными: параболичность соответствующих уравнений Колмогорова переносится на условие роста символа с индексом Соболева $\alpha$. Показывается, что соответствующая эволюционная задача является параболической для пространства Соболева-Слободецкого $H^{\alpha / 2}$ тогда и только тогда, когда процесс имеет индекс Соболева $\alpha$. Мы соотносим индекс Соболева с индексом Блюменталя-Гетура. Это показывает, что индекс Соболева является индикатором как гладкости распределения, так и вариации траектории процесса. Приводятся различные примеры процессов Леви с индексом Соболева и без него. В заключение мы демонстрируем влияние индекса Соболева на работу численных схем решения краевых задач, соответствующих процессам CGMY (Carr-Geman-Madan-Yor).

Ключевые слова и фразы: процессы Леви, интегро-дифференциальные уравнения с частными производными, символ процесса Леви, слабые решения, параболическое эволюционное уравнение, пространства Соболева-Слободецкого, метод Галёркина, расчет опциона.
\end{abstract}

1. Introduction. The Feynman-Kac formula provides a fundamental link between conditional expectations and solutions to partial differential equations. Under suitable regularity assumptions, a Feynman-Kac representation relates conditional expectations to solutions of Kolmogorov equations. In financial mathematics this fact is used to compute option prices by solving parabolic Kolmogorov equations.

*Technische Universität München, Garching, Germany, e-mail: kathrin.glau@tum.de

1) The author expresses her gratitude to Ernst Eberlein for his valuable support over the years. The author further thanks Christoph Schwab for fruitful discussions and thanks him and his working group at ETH Zürich for the MATLAB code for pricing a call option in a CGMY model based on a wavelet-Galerkin scheme. The author further thanks Antonis Papapantoleon, Mathias Beiglböck and especially Claudia Klüppelberg for critical reading and for fruitful comments on the manuscript and thanks Jean Jacod for helpful discussions. The author thanks Carsten Eilks for his great support. The support by the DFG through project EB66/11-1 is gratefully acknowledged. 
In the context of Lévy processes, conditional expectations are linked to solutions of Partial Integro-Differential Equations (PIDEs). WaveletGalerkin methods for pricing European and American options have been developed in [16], [15], [14]. Recent developments in P(I)DE techniques in finance are deeply and broadly discussed in [12]. For finite element methods the existence and uniqueness of a variational solution of PIDEs in a Sobolev-Slobodeckii space is essential.

The classical result on existence and uniqueness of variational solutions can be formulated as follows. Let $\left(V, H, V^{*}\right)$ be a Gelfand triplet, i.e., $V$ and $H$ are Hilbert spaces such that there exist continuous embeddings from $V$ into $H$ and from $H$ into the dual space $V^{*}$ of $V$, see [24, section 17.1]. Let $a: V \times V \rightarrow \mathbb{C}$ be a bilinear form that is continuous and satisfies a Garding inequality, i.e., there exist constants $C_{1}, C_{2}>0$ and $C_{3} \geqslant 0$ such that

$$
\begin{aligned}
|a(u, v)| & \leqslant C_{1}\|u\|_{V}\|v\|_{V} & & \text { (continuity) } \\
\operatorname{Re}(a(u, u)) & \geqslant C_{2}\|u\|_{V}^{2}-C_{3}\|u\|_{H}^{2} & & \text { (Gårding inequality), }
\end{aligned}
$$

for every $u, v \in V$. Then we call the evolution problem

$$
\dot{u}+\mathscr{A} u=f, \quad u(0)=g
$$

with $f \in L^{2}\left(0, T ; V^{*}\right)$ and $g \in H$ parabolic with respect to the Gelfand triplet $\left(V, H, V^{*}\right)$.

Hence, operator $\mathscr{A}$ of (1.3) and bilinear form $a$ of (1.1) are associated via $\mathscr{A} u:=a(u, \cdot): V \rightarrow \mathbb{C}$. The result itself can now be phrased as follows, cf., e.g., [24, Theorem 26.1] and [25, Theorem 23.A].

Theorem 1.1. Let the evolution problem (1.3) be parabolic with respect to the Gelfand triplet $\left(V, H, V^{*}\right)$. Then, it has a unique variational or weak solution $u \in W^{1}(0, T ; V, H)$. Moreover, the solution $u$ depends continuously on the data $g$ and $f$.

The proof of this theorem is based on a so-called Galerkin-approximation that yields a numerical scheme to calculate the solution approximately, namely a finite element scheme, see, e.g., [25, Theorem 23.A]. For the precise definition of a weak solution and of the space $W^{1}(0, T ; V, H)$ we refer to $[24$, section 25].2)

The Kolmogorov equations we are interested in are of type (1.3) where the operator $-\mathscr{A}$ is the infinitesimal generator of a Lévy process. Let $L$

2) The space $W^{1}(0, T ; V, H)$ consists of those functions $u \in L^{2}(0, T ; V)$ that have a derivative $\dot{u}$ with respect to time in a distributional sense that belongs to the space $L^{2}\left(0, T ; V^{*}\right)$. For a Hilbert space $H$, the space $L^{2}(0, T ; H)$ denotes the space of functions $u:[0, T] \rightarrow H$, that are weakly measurable and that satisfy $\int_{0}^{T}\|u(t)\|_{H}^{2} \mathrm{~d} t<\infty$. For the definition of weak measurability and for a detailed introduction of the space $W^{1}(0, T ; V, H)$ that relies on the Bochner integral, we refer to [24, section 24.2]. 
be an $\mathbb{R}^{d}$-valued Lévy process with characteristics $(b, \sigma, F)$ with respect to a truncation function $h$. Here, a measurable function $h: \mathbb{R}^{d} \rightarrow \mathbb{R}$ is called a truncation function if $\int_{\{|x|>1\}} h(x) F(\mathrm{~d} x)<\infty$ and $h(x)=x$ in a neighborhood of 0 . The distribution of the process is uniquely determined by the distribution $P^{L_{t}}$ for any $t>0$ and hence by the characteristic function of $L_{t}$,

$$
\mathbf{E} \mathrm{e}^{i\left\langle\xi, L_{t}\right\rangle}=\mathrm{e}^{-t A(-\xi)}
$$

where the symbol $A$ of the process is given by

$$
A(\xi):=\frac{1}{2}\langle\xi, \sigma \xi\rangle+i\langle\xi, b\rangle-\int\left(\mathrm{e}^{-i\langle\xi, y\rangle}-1+i\langle\xi, h(y)\rangle\right) F(\mathrm{~d} y)
$$

compare, e.g., [13, equation (0.9)]. By its defining properties one may show that there is a positive constant $C>0$ such that

$$
|A(\xi)| \leqslant C(1+|\xi|)^{2} \quad \text { for every } \xi \in \mathbb{R}^{d} .
$$

Denoting by $\widehat{u}$ the Fourier transform of $u$, by inequality (1.5) we have $A \widehat{u} \in$ $L^{2}\left(\mathbb{R}^{d}\right) \cap L^{1}\left(\mathbb{R}^{d}\right)$ for every function $u \in C_{0}^{\infty}\left(\mathbb{R}^{d}\right)$ and the Fourier inverse of $A \widehat{u}$ is well defined. Moreover, an elementary calculation shows that for

$$
\begin{aligned}
\mathscr{A} u(x):= & -\frac{1}{2} \sum_{j, k=1}^{d} \sigma^{j, k} \frac{\partial^{2} u}{\partial x_{j} \partial x_{k}}(x)-\sum_{j=1}^{d} b^{j} \frac{\partial u}{\partial x_{j}}(x) \\
& -\int_{\mathbb{R}^{d}}\left(u(x+y)-u(x)-\sum_{j=1}^{d} \frac{\partial u}{\partial x_{j}}(x)(h(y))_{j}\right) F(\mathrm{~d} y)
\end{aligned}
$$

we have

$$
\mathscr{A} u(x)=\frac{1}{(2 \pi)^{d}} \int A(\xi) \widehat{u}(\xi) \mathrm{e}^{-i\langle x, \xi\rangle} \mathrm{d} \xi
$$

for every $x \in \mathbb{R}^{d}$ and every $u \in C_{0}^{\infty}\left(\mathbb{R}^{d}\right)$. Equation (1.7) coincides with the definition of a pseudo differential operator $(P D O) \mathscr{A}$ with symbol $A$. It follows from the Parseval identity, see, e.g., [20, equality (10) on p. 187], that the bilinear form $a$ is given by

$$
\begin{aligned}
a(u, v) & :=\int \mathscr{A} u(x) v(x) \mathrm{d} x \\
& =\frac{1}{(2 \pi)^{d}} \int_{\mathbb{R}^{d}} A(\xi) \widehat{u}(\xi) \overline{\widehat{v}(\xi)} \mathrm{d} \xi \quad \text { for all } u, v \in C_{0}^{\infty}\left(\mathbb{R}^{d}\right) .
\end{aligned}
$$

While PIDEs are classified via their operators, Lévy processes are determined by their characteristic functions due to the famous Lévy-Khinchin formula. Various classes of Lévy processes, such as tempered stable processes, are even directly defined by their characteristic functions. 
Our general question therefore is: Under which conditions on the symbol is the Kolmogorov equation parabolic? The answer is given in Theorem 2.1.

In order to solve parabolic equations numerically, we rely on welldeveloped Galerkin methods. The solution space is crucial for the numerical scheme and its convergence rate. Thus, the relation between Lévy processes and solution spaces is relevant for both theoretical and numerical considerations. For theoretical purposes it is convenient to work with an explicitly given well-known solution space for which standard convergence results and inequalities are available. From a numerical perspective, the degree of regularity of the solution is the determining factor. Classically, the order of differentiability of the solution is directly related to the order of convergence of the finite element scheme, see, e.g., [22, Theorem 1.1]. Such a correspondence is still valid for approximation schemes for parabolic integro-differential equations. Figures 5.1 and 5.2 provide a numerical illustration of the role of the fractional degree of regularity to the performance of the scheme for Kolmogorov equations of a class of Lévy processes. See [23, Theorem 5.4] for theoretical convergence results. Here, fractional orders of differentiation appear naturally in the convergence estimates and the corresponding solution spaces are Sobolev-Slobodeckii spaces.

Hence, our second question is: Under which conditions on the symbol is the Kolmogorov equation parabolic with a Sobolev-Slobodeckii space as solution space? This leads us to the definition of a Sobolev index of a symbol and of the corresponding process, respectively.

It follows from [6, Theorem 6.1 and equation (31)] that if the Lévy process $L$ has a Sobolev index, then the corresponding weak solution $u$ of equation (1.3) for $f \equiv 0, g \in L^{2}(\mathbb{R})$, and $\mathscr{A}$ given by (1.6) has the FeynmanKac representation

$$
u\left(T-t, L_{t}\right)=\mathbf{E}\left[g\left(L_{T}\right) \mid \mathscr{F}_{t}\right] \quad \text { a.s. }
$$

Thus, our analysis of the parabolicity of the related Kolmogorov equations characterizes conditional expectations of functional transforms of Lévy processes by weak solutions. A more general result involving a killing rate is provided in [10]. A related reference is [4, Theorem 8.2], where Markovian semimartingales with Brownian parts stopped at their first exit out of a bounded domain are related by a Feynman-Kac formula to solutions of boundary value problems. These results show that under appropriate assumptions a variety of conditional expectations on functionals of Lévy processes are given by weak solutions to Kolmogorov equations. Our analysis yields appropriate conditions for the applicability of Galerkin methods for computing these expectations numerically.

Our paper is organized as follows: In section 2, we derive the general theorem on parabolicity of the Kolmogorov equations. This section demonstrates the general validity of the theory that is not related to Sobolev- 
Slobodeckii spaces only. We then define the Sobolev index of a symbol or of a Lévy process, respectively, in terms of polynomial growth conditions on the symbol of the process. We show that the corresponding evolution problem is of parabolic type with solution space $H^{\alpha / 2}$ if and only if the process has Sobolev index $\alpha$. Various examples are discussed in section 3. Since the existence of a Sobolev index is necessary and sufficient for the parabolicity of the related Kolmogorov backward equation with respect to Sobolev-Slobodeckii spaces, not every Lévy process has a Sobolev index. We show that if the Lévy process has Sobolev index $\alpha<2$, then $\alpha$ is the Blumenthal-Getoor index. Thereby a new link is established between path properties of the process and the smoothing effect of the related evolution problem. This link refines the connection between jump diffusion processes and the solution spaces of the related Kolmogorov equations established in [4]. This allows us to conclude for several examples that they do not have a Sobolev index. For a wide class of Lévy processes we show the existence of a Sobolev index and compute its value. We provide a tail condition and conditions on the density of the Lévy measure that imply the existence of a Sobolev index. A numerical illustration of the effect of the Sobolev index on the performance of numerical schemes is provided in the last section. The analysis shows a higher regularity of the solution for higher Sobolev indices of the underlying driving Lévy process. This suggests higher rates of convergence of the numerical schemes, which is confirmed by numerical experiments, where we use a wavelet-Galerkin scheme to numerically evaluate a boundary value problem related to a CGMY Lévy process for different parameters.

2. Parabolicity of Kolmogorov equations. Notice that the real part of the symbol of a Lévy process is nonnegative,

$$
\operatorname{Re}(A(\xi))=\langle\xi, \sigma \xi\rangle-\int(\cos \langle x, \xi\rangle-1) F(\mathrm{~d} y) \geqslant 0 .
$$

We define the Hilbert space $H^{\operatorname{Re}(A)+k}$ for $k \geqslant 1$ as the completion of $C_{0}^{\infty}\left(\mathbb{R}^{d}, \mathbb{R}\right)$, i.e., of the real-valued smooth functions with compact support in $\mathbb{R}^{d}$, with respect to the norm $\|\cdot\|_{\operatorname{Re}(A)+k}$ given by

$$
\|u\|_{\operatorname{Re}(A)+k}:=\frac{1}{(2 \pi)^{d}} \int_{\mathbb{R}^{d}}(k+\operatorname{Re}(A(\xi)))|\widehat{u}(\xi)|^{2} \mathrm{~d} \xi,
$$

and denote by $\left(H^{\operatorname{Re}(A)+k}\right)^{*}$ the dual space of $H^{\operatorname{Re}(A)+k}$. For $u \in C_{0}^{\infty}\left(\mathbb{R}^{d}, \mathbb{R}\right)$ it follows from (1.8) that

$$
a(u, u)=\frac{1}{(2 \pi)^{d}} \int_{\mathbb{R}^{d}} A(\xi)|\widehat{u}(\xi)|^{2} \mathrm{~d} \xi=\frac{1}{(2 \pi)^{d}} \int_{\mathbb{R}^{d}} \operatorname{Re}(A(\xi))|\widehat{u}(\xi)|^{2} \mathrm{~d} \xi
$$

and hence

$$
a(u, u)=\|u\|_{\operatorname{Re}(A)+k}^{2}-k\|u\|_{L^{2}}^{2} .
$$


We obtain the following result.

Theorem 2.1. Let $A$ be the symbol of the Lévy process L. Assume

$$
|\operatorname{Im}(A(\xi))| \leqslant c(k+\operatorname{Re}(A(\xi))) \quad \text { for all } \xi \in \mathbb{R}^{d}
$$

with some positive constant $c \geqslant 0$. Then, the bilinear form a is continuous and satisfies a Gärding inequality with respect to the norms of $H^{\operatorname{Re}(A)+k}$ and $L^{2}\left(\mathbb{R}^{d}\right)$, hence, the evolution problem

$$
\dot{u}+\mathscr{A} u=f, \quad u(0)=g
$$

with $f \in L^{2}\left(0, T ;\left(H^{\operatorname{Re}(A)+k}\left(\mathbb{R}^{d}\right)\right)^{*}\right)$ and initial condition $g \in L^{2}\left(\mathbb{R}^{d}\right)$ is parabolic with respect to the Gelfand triplet $\left(H^{\operatorname{Re}(A)+k}, L^{2}\left(\mathbb{R}^{d}\right),\left(H^{\operatorname{Re}(A)+k}\right)^{*}\right)$ and has a unique variational solution $u \in W^{1}\left(0, T ; H^{\operatorname{Re}(A)+k}, L^{2}\left(\mathbb{R}^{d}\right)\right)$.

P r o o f. Since $C_{0}^{\infty}\left(\mathbb{R}^{d}, \mathbb{R}\right)$ is dense in $H^{\operatorname{Re}(A)+k}$, it follows from [24, Theorem 17.1 and Definition 17.1] that $\left(H^{\operatorname{Re}(A)+k}, L^{2}\left(\mathbb{R}^{d}\right),\left(H^{\operatorname{Re}(A)+k}\right)^{*}\right)$ is a Gelfand triplet. Equality (2.2) implies the Gårding inequality

$$
\operatorname{Re}(a(u, u)) \geqslant\|u\|_{\operatorname{Re}(A)+k}^{2}-k\|u\|_{L^{2}}^{2}
$$

for every $u \in C_{0}^{\infty}\left(\mathbb{R}^{d}, \mathbb{R}\right)$. From assumption (2.3), we obtain for $u, v \in$ $C_{0}^{\infty}\left(\mathbb{R}^{d}, \mathbb{R}\right)$

$$
\begin{aligned}
|a(u, v)| & \leqslant(1+c) \int_{\mathbb{R}^{d}}|k+\operatorname{Re}(A(\xi))||\widehat{u}(\xi) \overline{\widehat{v}(\xi)}| \mathrm{d} \xi \\
& \leqslant(1+c)\|u\|_{\operatorname{Re}(A)+k}\|v\|_{\operatorname{Re}(A)+k} .
\end{aligned}
$$

The bilinear form thus has a unique continuous and linear extension $a: H^{\operatorname{Re}(A)+k} \times H^{\operatorname{Re}(A)+k} \rightarrow \mathbb{R}$ and the evolution problem is well defined. Furthermore, the Gårding inequality is also satisfied for the extension and it follows that the evolution problem is parabolic with respect to the Gelfand triplet $\left(H^{\operatorname{Re}(A)+k}, L^{2}\left(\mathbb{R}^{d}\right),\left(H^{\operatorname{Re}(A)+k}\right)^{*}\right)$. The existence and uniqueness of a variational solution then follows from the classical Theorem 1.1. Theorem 2.1 is proved.

Inequality (2.3) is slightly weaker than the so-called sector condition, $|\operatorname{Im}(A(\xi))| \leqslant c \operatorname{Re}(A(\xi))$ for some constant $c>0$ for all $\xi \in \mathbb{R}^{d}$. The bilinear form $a$ defines a so-called nonsymmetric Dirichlet form if and only if the sector condition is satisfied, see [5, Example 3.13] and the references therein.

$\mathrm{R}$ e $\mathrm{m}$ a $\mathrm{r} \mathrm{k}$ 2.1. Theorem 2.1 is satisfactory from an abstract point of view solely. With regards to convergence of numerical schemes, regularity of the solutions is crucial. Notice that for symmetric Poisson processes, to mention a simple example, the symbol is real, positive and bounded. In particular inequality (2.3) is satisfied for every $k \geqslant 0$. Moreover, the space $H^{\operatorname{Re}(A)+k}(\mathbb{R})$ coincides with $L^{2}(\mathbb{R})$ and the norms of both spaces are 
equivalent. Thus, the parabolic problem is degenerated in the sense that it has no smoothing effect.

In the following, we focus on the case that the space $H^{\operatorname{Re}(A)+k}$ is a Sobolev-Slobodeckii space, compare Remark 3.1. A major advantage of these concrete spaces is that they describe regularity in the sense that a fractional order derivative exists and is in $L^{2}$.

3. Parabolicity of Kolmogorov equations with respect to Sobolev-Slobodeckii spaces. Let us denote by $S\left(\mathbb{R}^{d}\right)$ the Schwartz space, i.e., the set of complex-valued smooth functions $\varphi \in C^{\infty}\left(\mathbb{R}^{d}, \mathbb{C}\right)$ with

$$
\left(1+|x|^{m}\right)\left|D^{\alpha} \varphi(x)\right| \rightarrow 0, \quad|x| \rightarrow \infty
$$

for every multiindex $\alpha=\left(\alpha_{1}, \ldots, \alpha_{d}\right) \in \mathbb{N}_{0}^{d}$ and every $m \in \mathbb{N}_{0}$, where $D^{\alpha}$ denotes the multiple partial derivative

$$
D^{\alpha} \varphi(x):=\frac{\partial^{\alpha_{1}} \cdots \partial^{\alpha_{d}}}{\partial x_{1}^{\alpha_{1}} \cdots \partial x_{d}^{\alpha_{d}}} \varphi(x) .
$$

We denote by $S^{\prime}\left(\mathbb{R}^{d}\right)$ the space of generalized functions, i.e., the dual space of the Schwartz space $S\left(\mathbb{R}^{d}\right)$. By duality, one defines the Fourier transform $\widehat{u}$ of a generalized function $u$. Moreover, denote

$$
\|u\|_{s}^{2}:=\int|\widehat{u}(\xi)|^{2}(1+|\xi|)^{2 s} \mathrm{~d} \xi
$$

Sobolev-Slobodeckii spaces are defined by

$$
H^{s}\left(\mathbb{R}^{d}\right):=\left\{u \in S^{\prime}\left(\mathbb{R}^{d}\right) \mid \widehat{u} \in L_{\text {loc }}^{1}\left(\mathbb{R}^{d}, \mathbb{C}^{d}\right) \text { with }\|u\|_{s}^{2}<\infty\right\}
$$

for $s \in \mathbb{R}$. It is shown in [24, Definition 5.1] that for any $s \in \mathbb{R}, H^{s}\left(\mathbb{R}^{d}\right)$ is a Hilbert space. Moreover, Eskin [8, section I.4.5] has shown that the dual space of $H^{s}\left(\mathbb{R}^{d}\right)$ may be identified with the space $H^{-s}\left(\mathbb{R}^{d}\right)$. Wloka [24, Example 17.1] shows that $\left(H^{s}\left(\mathbb{R}^{d}\right), L^{2}\left(\mathbb{R}^{d}\right), H^{-s}\left(\mathbb{R}^{d}\right)\right)$ is a Gelfand triplet.

$\mathrm{D}$ e f i n it i o n 3.1. Let $\mathscr{A}$ be a pseudo differential operator with symbol $A$. We say $\alpha \in(0,2]$ is the Sobolev index of the symbol $A$, if for all $\xi \in \mathbb{R}^{d}$

$$
\begin{aligned}
|A(\xi)| & \leqslant C_{1}\left(1+|\xi|^{2}\right)^{\alpha / 2} & & \text { (continuity condition) } \\
\operatorname{Re}(A(\xi)) & \geqslant C_{2}|\xi|^{\alpha}-C_{3}\left(1+|\xi|^{2}\right)^{\beta / 2} & & \text { (Gårding condition) }
\end{aligned}
$$

for some $0 \leqslant \beta<\alpha$ and constants $C_{1}, C_{3} \geqslant 0$ and $C_{2}>0$.

If $L$ is a Lévy process with symbol $A$ and Sobolev index $\alpha$, we call $\alpha$ the Sobolev index of the Lévy process $L$. 
Theorem 3.1. Let $\mathscr{A}$ be a pseudo differential operator with symbol $A$ and bilinear form $a$. Then assertion (i) below implies assertion (ii). Moreover, if the symbol $A$ is continuous, then assertions (i) and (ii) are equivalent.

(i) The symbol $A$ has a Sobolev index $\alpha \in(0,2]$.

(ii) The bilinear form a satisfies continuity and a Gärding inequality with respect to the norms of $H^{\alpha / 2}$ and $L^{2}$. Hence, the evolution problem

$$
\dot{u}+\mathscr{A} u=f, \quad u(0)=g
$$

with $f \in L^{2}\left(0, T ; H^{-s}\left(\mathbb{R}^{d}\right)\right)$ and initial condition $g \in L^{2}\left(\mathbb{R}^{d}\right)$ is parabolic with respect to the Gelfand triplet $\left(H^{\alpha / 2}\left(\mathbb{R}^{d}\right), L^{2}\left(\mathbb{R}^{d}\right), H^{-\alpha / 2}\left(\mathbb{R}^{d}\right)\right)$ and has a unique variational solution $u \in W^{1}\left(0, T ; H^{\alpha / 2}\left(\mathbb{R}^{d}\right), L^{2}\left(\mathbb{R}^{d}\right)\right)$.

$\mathrm{P}$ r o o f. The proof of implication (i) $\Rightarrow$ (ii) is provided in [12]. It follows from standard analysis arguments using the following inequalities: For $C_{1}>0, C_{2} \geqslant 0,0 \leqslant \beta<\alpha$ and $0<C_{3}<C_{1}$ there exits a constant $C_{4}>0$ such that $C_{1} x^{\alpha}-C_{2} x^{\beta} \geqslant C_{3} x^{\alpha}-C_{4}$ for every $x \geqslant 0$ and

$$
C_{2}|\xi|^{2 \alpha}-C_{3}\left(1+|\xi|^{2}\right)^{\beta} \geqslant C_{2}|\xi|^{2 \alpha}-C_{3}^{\prime}\left(1+|\xi|^{2 \beta}\right) \geqslant c_{2}(1+|\xi|)^{2 \alpha}-c_{3}
$$

with a strictly positive constant $c_{2}$ and $C_{3}^{\prime}, c_{3} \geqslant 0$.

In order to derive implication (ii) $\Rightarrow$ (i) for continuous symbols $A$, we closely follow the derivation of the fundamental lemma of variational calculus.

To this end, we extend the domain of the bilinear form, which is possible thanks to the continuity condition (3.1). We now call $a$ the unique continuous bilinear form $a: H^{\alpha / 2}\left(\mathbb{R}^{d}\right) \times H^{\alpha / 2}\left(\mathbb{R}^{d}\right) \rightarrow \mathbb{C}$ that, restricted to the compactly supported smooth functions, coincides with the bilinear form $a$ given in the theorem. The continuity of the bilinear form yields

$$
\begin{array}{ll}
\int_{\mathbb{R}^{d}}\left(c_{1}(1+|\xi|)^{\alpha} \pm \operatorname{Re}(A(\xi))\right)|\widehat{u}(\xi)|^{2} \mathrm{~d} \xi \geqslant 0 & \text { for any } u \in H^{\alpha / 2}\left(\mathbb{R}^{d}\right), \\
\int_{\mathbb{R}^{d}}\left(c_{1}(1+|\xi|)^{\alpha} \pm \operatorname{Im}(A(\xi))\right)|\widehat{u}(\xi)|^{2} \mathrm{~d} \xi \geqslant 0 & \text { for any } u \in H^{\alpha / 2}\left(\mathbb{R}^{d}\right) .
\end{array}
$$

Let us now assume the continuity condition would not be satisfied. Then, one out of the four integrands would become nonnegative for some $\xi$. Thanks to the continuity of the symbol, the integrand would be negative on a nonempty open subset of $U \subset \mathbb{R}^{d}$. We may choose a function $u$ such that its Fourier transform $\widehat{u}$ is smooth, not constant and such that its compact support is contained in $U$. Noting that those functions lie in the Schwartz space which is contained in $H^{\alpha / 2}\left(\mathbb{R}^{d}\right)$, we would obtain a contradiction.

Moreover, the Gårding inequality is satisfied on the extended domain which yields

$$
\int_{\mathbb{R}^{d}}\left(\operatorname{Re}(A(\xi))-C_{1}(1+|\xi|)^{\alpha}+C_{2}\right)|\widehat{u}(\xi)|^{2} \mathrm{~d} \xi \geqslant 0 \quad \text { for any } u \in H^{\alpha / 2}\left(\mathbb{R}^{d}\right) .
$$


Arguing along the same lines as for the continuity, we obtain that the Gårding condition is satisfied. Theorem 3.1 is proved.

Corollary 3.1. (i) In case of continuity of $\xi \mapsto A(\xi)$, the Garding condition (3.2) is equivalent to the existence of constants $C_{2}>0$ and $N \geqslant 0$ such that

$$
\operatorname{Re}(A(\xi)) \geqslant C_{2}|\xi|^{\alpha} \quad \text { for all }|\xi|>N
$$

and is equivalent to the existence of constants $C_{2}>0$ and $C_{3} \geqslant 0$ such that

$$
\operatorname{Re}(A(\xi)) \geqslant C_{2}|\xi|^{\alpha}-C_{3} \text { for all } \xi \in \mathbb{R}^{d} .
$$

(ii) Let $L$ be a Lévy process. Then its symbol is continuous and the Gårding condition (3.2) is equivalent to inequality (3.4). Moreover, the existence of a Sobolev index $\alpha \in(0,2]$ is equivalent to the parabolicity of the Kolmogorov equation (3.3) with respect to the Gelfand triplet $\left(H^{\alpha / 2}\left(\mathbb{R}^{d}\right)\right.$, $\left.L^{2}\left(\mathbb{R}^{d}\right), H^{-\alpha / 2}\left(\mathbb{R}^{d}\right)\right)$.

$\mathrm{R}$ e m a r k 3.1. For continuous symbols $A$, the Garding condition (3.2) implies by (3.5) the existence of a constant $k \geqslant 1$ such that $H^{\operatorname{Re}(A)+k}\left(\mathbb{R}^{d}\right)$ from section 2 coincides with $H^{\alpha / 2}\left(\mathbb{R}^{d}\right)$ and the norms of the spaces are equivalent. If additionally the continuity condition (3.1) is satisfied, (2.3) is also valid.

We generalize assertion (ii) of Theorem 3.1 to boundary value problems. The weak formulation of the boundary value problem on a domain $D$ with zero boundary condition is based on the space $\widetilde{H}^{\alpha / 2}(D)$ which is the space of functions in $H^{\alpha / 2}\left(\mathbb{R}^{d}\right)$ that vanish outside of $D$. More precisely, for $D=\mathbb{R}^{d}$, the space $\widetilde{H}^{\alpha / 2}(D)$ coincides with the Sobolev-Slobodeckii space $H^{\alpha / 2}\left(\mathbb{R}^{d}\right)$ and for $D \subset \mathbb{R}^{d}$ open, it denotes the space of generalized functions $u \in$ $H^{\alpha / 2}\left(\mathbb{R}^{d}\right)$ with $\left.u\right|_{D^{c}} \equiv 0$ in distributional sense. Moreover, we denote by $\left(\widetilde{H}^{s}(D)\right)^{*}$ the dual space of $\widetilde{H}^{s}(D)$.

Let $A$ be a symbol of operator $\mathscr{A}$ with Sobolev index $\alpha$ for some $\alpha>0$, and consider the associated bilinear form $a: H^{s}\left(\mathbb{R}^{d}\right) \times H^{s}\left(\mathbb{R}^{d}\right) \rightarrow \mathbb{R}$. Since $\widetilde{H}^{\alpha / 2}(D)$ is a subspace of $H^{\alpha / 2}\left(\mathbb{R}^{d}\right)$, the bilinear forms $a:=\left.a\right|_{\widetilde{H}^{\alpha / 2}(D) \times \widetilde{H}^{\alpha / 2}(D)}$ and $\mathscr{A}:=\left.\mathscr{A}\right|_{\widetilde{H}^{s}(D)}$ are well defined. Crucial for the following theorem is that the space of smooth functions with compact support is dense in $\widetilde{H}^{\alpha / 2}(D)$. This is the case under the so-called segment property for the domain $D$, which is provided in [24, Definition 2.1].

Corollary 3.2. Let $\mathscr{A}$ be a pseudo differential operator with symbol $A$ and Sobolev index $\alpha$ for some $\alpha>0$. Let further $D \subset \mathbb{R}^{d}$ be an open set satisfying the segment property. Then the parabolic equation

$$
\partial_{t} u+\mathscr{A} u=f, \quad u(0)=g,
$$

for $f \in L^{2}\left(0, T ; \widetilde{H}^{-\alpha / 2}(D)\right)$ and initial condition $g \in L^{2}(D)$ has a unique weak solution $u$ in the space $W^{1}\left(0, T ; \widetilde{H}^{\alpha / 2}(D), L^{2}(D)\right)$. 
P r o o f. For $s \geqslant 0, u \in H^{s}\left(\mathbb{R}^{d}\right)$, and $\varphi \in S\left(\mathbb{R}^{d}\right)$ we denote by $\mathscr{F}$ the Fourier transform and by $\mathscr{F}^{-1}$ its inverse. Furthermore, we denote by $i$ the isomorphism that identifies a generalized function with its $L^{2}$-Riesz representative on its domain. From the Parseval identity we get

$$
\begin{aligned}
(u, \varphi) & =\frac{1}{(2 \pi)^{d}}(\mathscr{F}(u), \mathscr{F}(\varphi))=\frac{1}{(2 \pi)^{d}} \int i^{-1} \mathscr{F}(u)(\xi) \overline{\mathscr{F}(\varphi)}(\xi) \mathrm{d} \xi \\
& =\int \mathscr{F}^{-1}\left(i^{-1} \mathscr{F}(u)\right)(\xi) \overline{\varphi(\xi)} \mathrm{d} \xi
\end{aligned}
$$

For $s \geqslant 0$, Lemma 4.1 in [8] shows that the norm $\|\cdot\|_{s}$ is equivalent to the norm $\|\cdot\|_{s}^{\prime}$ given by

$$
\|u\|_{s}^{\prime}=\sum_{|l|=k} \int_{\mathbb{R}^{d}} \int_{\mathbb{R}^{d}} \frac{\left|D^{l} u(x+y)-D^{l} u(x)\right|^{2}}{|y|^{d+2 \lambda}} \mathrm{d} x \mathrm{~d} y+\int|u(x)|^{2} \mathrm{~d} x
$$

for $k \in \mathbb{N}_{0}$ and $\lambda \in(0,1)$ such that $s=k+\lambda$.

Thus, $\widetilde{H}^{s}(D)$ is equivalent to the space of complex-valued square integrable functions $u \in L^{2}\left(\mathbb{R}^{d}, \mathbb{C}\right)$ with $\|u\|_{s}^{\prime}<\infty$ that vanish almost surely on $D^{c}$. In particular, up to isomorphy we have

$$
\widetilde{H}^{s}(D) \subset W^{s}(D):=\left\{u \in L^{2}(D, \mathbb{C}) \mid\|u\|_{s}^{\prime}<\infty\right\} .
$$

Since $D$ satisfies the segment property, Theorem 3.6 in [24] shows that $C_{0}^{\infty}(D, \mathbb{C})$, the set of smooth complex functions with compact support in $D$, is dense in $W^{s}(D)$ and hence also in $\widetilde{H}^{s}(D)$.

Moreover, there exists an embedding $\widetilde{H}^{s}(D) \hookrightarrow L^{2}(D)$ which is continuous and injective. Since $C_{0}^{\infty}(D, \mathbb{C})$ is dense in $L^{2}(D)$ and $C_{0}^{\infty}(D) \subset \widetilde{H}^{s}(D)$, the density of $\widetilde{H}^{s}(D)$ in $L_{\eta}^{2}(D)$ follows. Since $\widetilde{H}^{s}(D)$ is a Hilbert space, [24, Theorem 17.1 and Definition 17.1] yield that $\left(\widetilde{H}^{\alpha / 2}(D), L^{2}(D),\left(\widetilde{H}^{\alpha / 2}(D)\right)^{*}\right)$ is a Gelfand triplet.

The bilinear form $a$, associated to $\mathscr{A}$ in equation (3.6), is the restriction of $a: H^{\alpha / 2}(\mathbb{R}) \times H^{\alpha / 2}\left(\mathbb{R}^{d}\right) \rightarrow \mathbb{R}$ to $\widetilde{H}^{\alpha / 2}(D) \times \widetilde{H}^{\alpha / 2}(D)$. Hence, the continuity and Gårding inequality carry over from the original bilinear form. Corollary 3.2 is proved.

We now discuss the existence of smooth densities of Lévy processes. The existence of a smooth density is discussed in [17] under the condition that the Lévy measure $F$ satisfies $\liminf _{r \downarrow 0} r^{\alpha-2} \int_{[-r, r]}|x|^{2} F(\mathrm{~d} x)>0$. We will prove in Proposition 4.1 that this condition implies the Gårding condition (3.2). Part (i) of the following proposition shows the existence of a smooth density under the Gårding condition (3.2). Part (ii) shows the existence of smooth densities for Lévy processes whose Kolmogorov equations are parabolic with respect to Sobolev-Slobodeckii spaces. 
Proposition 3.1. Let $L$ be Lévy process with symbol $A$ and bilinear form a. Then, for every $t>0$ the distribution of $L_{t}$ has a smooth and bounded density with respect to the Lebesgue measure if one of the following conditions holds:

(i) The symbol A satisfies Gärding condition (3.2) for some $\alpha \in(0,2]$;

(ii) the bilinear form a satisfies continuity condition and a Gärding inequality with respect to the norms of $H^{\alpha / 2}$ and $L^{2}$.

P r o o f. (i) The Fourier transform of the measure $\mu_{t}$ is given by $\widehat{\mu}_{t}(\xi)=$ $\mathrm{e}^{-t A(-\xi)}$ and

$$
\left|\widehat{\mu}_{t}(\xi)\right|=\mathrm{e}^{-t \operatorname{Re}(A(-\xi))} \leqslant \mathrm{e}^{-C_{2} t|\xi|^{\alpha}+C_{3} t\left(1+|\xi|^{2}\right)^{\beta / 2}}
$$

with $C_{2}>0, C_{3} \geqslant 0$, and $0 \leqslant \beta<\alpha$ by assumption. This shows that the term $\left|\widehat{\mu}_{t}(\xi)\right|$ decays exponentially fast as $|\xi| \rightarrow \infty$. Together with the continuity of $\xi \mapsto \operatorname{Re}(A(\xi))$ finiteness of the moments $\int_{\mathbb{R}^{d}}|\xi|^{n}\left|\hat{\mu}_{t}(\xi)\right| \mathrm{d} \xi<\infty$ for every $n \in \mathbb{N}$ follows. The assertion now follows from [21, Proposition 28.1].

(ii) The assertion is an immediate consequence of Theorem 3.1 and part (i) of this Proposition. Proposition 3.1 is proved.

$\mathrm{R}$ e $\mathrm{m}$ a r k 3.2. In light of Corollary 3.2, the Sobolev index appears as a measure of the degree of the smoothing effect of the related evolution problem on the solution. Under appropriate additional assumptions, a Feynman-Kac formula for weak solutions yields a stochastic representation: the conditional expectation of functionals of the path of the Lévy process are solutions of the PIDE. This can be interpreted as a smoothing effect of the distribution of the Lévy process.

This is worth mentioning, since it is much more involved to observe effects of the distribution of the process $\left(L_{t}\right)_{t \geqslant 0}$ than of the marginals $L_{t}$ for fixed times $t \geqslant 0$. For an appropriate Feynman-Kac formula see [9, Theorem IV.9].

4. Examples: Sobolev indices of Lévy processes. We relate the Sobolev index to the Blumenthal-Getoor index and give some examples of Lévy processes without Sobolev index. For several important classes of processes we will show that they have a Sobolev index and we compute its value.

4.1. Blumenthal-Getoor index and some Lévy processes without Sobolev index. For a Lévy process with characteristics $(b, c, F)$

$$
\beta:=\inf \left\{\gamma>\left.0\left|\int_{[-1,1]}\right| x\right|^{\gamma} F(\mathrm{~d} x)<\infty\right\} \leqslant 2
$$

is called the Blumenthal-Getoor index of the process, see, e.g., [21, p. 362].

Theorem 4.1. Let $L$ be a real-valued Lévy process with characteristics $(b, 0, F)$ and Sobolev index $0<\alpha<2$. Then its Sobolev index is equal to its Blumenthal-Getoor index, $\alpha=\beta$. 
P r o o f. Since the symmetric part of the Lévy measure around the origin determines the Blumenthal-Getoor index, we may assume without loss of generality that $F$ is symmetric with support in $[-1,1]$.

We show the assertion by deriving

$$
\begin{array}{ll}
\int|x|^{\alpha-\varepsilon} F(\mathrm{~d} x)=\infty & \text { for all } \varepsilon>0, \\
\int|x|^{\alpha+\varepsilon} F(\mathrm{~d} x)<\infty & \text { for all } 0 \leqslant \varepsilon<\alpha .
\end{array}
$$

From the assumption we know that there exist constants $C_{1}>0, C_{2} \geqslant 0$ and indices $0<\alpha^{\prime}<\alpha<2$ such that

$$
\int(1-\cos (u x)) F(\mathrm{~d} x) \geqslant C_{1}|u|^{\alpha}-C_{2}\left(1+|u|^{\alpha^{\prime}}\right) .
$$

Since for every $0<\gamma<2$ there exists a constant $C(\gamma)>0$ such that

$$
1-\cos y \leqslant C(\gamma)|y|^{\gamma}
$$

for all $y \in \mathbb{R}$, we are able to conclude for any fixed $\varepsilon>0$ that

$$
C_{1}|u|^{\alpha}-C_{2}|u|^{\alpha^{\prime}}-C_{\varepsilon} \leqslant \int_{-\varepsilon}^{\varepsilon}(1-\cos (u x)) F(\mathrm{~d} x) \leqslant C(\gamma) \int_{-\varepsilon}^{\varepsilon}|u x|^{\gamma} F(\mathrm{~d} x)
$$

with constant $C_{\varepsilon}=C_{2}+2 F\left((-\varepsilon, \varepsilon)^{c}\right)>0$ for all $u \in \mathbb{R}$, hence,

$$
\frac{C_{1}}{C(\gamma)}|u|^{\alpha-\gamma}-\frac{C_{2}}{C(\gamma)}|u|^{\alpha^{\prime}-\gamma}-\frac{C_{\varepsilon}}{C(\gamma)}|u|^{-\gamma} \leqslant \int_{-\varepsilon}^{\varepsilon}|x|^{\gamma} F(\mathrm{~d} x)
$$

for all $u \in \mathbb{R} \backslash\{0\}$. For every $\varepsilon>0$ the left-hand side of the inequality diverges for $|u| \rightarrow \infty$, if $\gamma<\alpha$. Thus we can conclude $\infty=\int_{-\varepsilon}^{\varepsilon}|x|^{\gamma} F(\mathrm{~d} x)$ for every $\varepsilon>0$ and $\gamma<\alpha$ which yields equation (4.1).

In order to show (4.2), we set

$$
\varphi_{\gamma}(x):=C_{\gamma}|x|^{\gamma} \mathrm{e}^{-|x|}
$$

with $C_{\gamma}:=\left(\int|x|^{\gamma} \mathrm{e}^{-|x|} \mathrm{d} x\right)^{-1}$ for each $0<\gamma<2$. Its Fourier transform is given by

$$
\widehat{\varphi}_{\gamma}(u)= \begin{cases}\Gamma(\gamma+1)\left(1+u^{2}\right)^{-(\gamma+1) / 2} \sin ((\gamma+1) \arctan u), & \text { for } \gamma \neq 1 \\ 2\left(1+u^{2}\right)^{-2}|u|, & \text { for } \gamma=1,\end{cases}
$$

where $\Gamma$ denotes the Gamma function. In particular, $\widehat{\varphi}_{\gamma}$ is real, symmetric, and integrable with $\varphi_{\gamma}(0)=0$, hence

$$
\varphi_{\gamma}(-x)=\frac{1}{2 \pi} \int\left(\mathrm{e}^{i u x}-1-i u h(x)\right) \widehat{\varphi}_{\gamma}(u) \mathrm{d} u=\frac{1}{2 \pi} \int(\cos (u x)-1) \hat{\varphi}_{\gamma}(u) \mathrm{d} u .
$$


Moreover,

$$
\int\left|\widehat{\varphi}_{\gamma}(u)\right|\left(1+u^{2}\right)^{(\gamma-\varepsilon) / 2} \mathrm{~d} u<\infty \quad \text { for every } \varepsilon>0
$$

It follows from the continuity condition (3.1) and from inequality (4.6) for $\gamma>\alpha$ that

$$
\begin{aligned}
\iint|\cos (u x)-1|\left|\widehat{\varphi}_{\gamma}(u)\right| F(\mathrm{~d} x) \mathrm{d} u & =\int \operatorname{Re}(A(u))\left|\hat{\varphi}_{\gamma}(u)\right| \mathrm{d} u \\
& \leqslant C_{1} \int\left(1+|u|^{\alpha}\right)\left|\widehat{\varphi}_{\gamma}(u)\right| \mathrm{d} u<\infty
\end{aligned}
$$

with some constant $C_{1}>0$. We obtain

$$
\begin{aligned}
\infty & >\int \widehat{\varphi}_{\gamma}(u) \operatorname{Re}(A(u)) \mathrm{d} u=\iint(1-\cos (u x)) F(\mathrm{~d} x) \widehat{\varphi}_{\gamma}(u) \mathrm{d} u \\
& =\iint(1-\cos (u x)) \widehat{\varphi}_{\gamma}(u) \mathrm{d} u F(\mathrm{~d} x)=C_{\gamma} \sqrt{2 \pi} \int|x|^{\gamma} \mathrm{e}^{-|x|} F(\mathrm{~d} x),
\end{aligned}
$$

where the second equality follows from the Fubini theorem and the last equality is due to equality (4.5). From a Taylor approximation around zero, assertion (4.2) follows. Theorem 4.1 is proved.

The following example shows that for a Lévy process $L$ with symbol $A$ and Blumenthal-Getoor index $\alpha \in(0,2)$, it is not sufficient to assume $|\operatorname{Im}(A(u))| \leqslant C\left(1+|u|^{2}\right)^{\alpha / 2}$ in order to guarantee that $\alpha$ is the Sobolev index of the process.

$\mathrm{Ex}$ a $\mathrm{m} \mathrm{ple} \mathrm{4.1.} \mathrm{Let} L$ be a Lévy process with characteristic triplet $(0,0, F)$ with Lévy density $F(\mathrm{~d} x):=-|x|^{-1-\alpha} \ln (|x|) \mathbb{I}_{[-1,1]}(x) \mathrm{d} x$. Then, there is a $0<\varepsilon^{\prime}<\min \{\mathrm{e}, \varepsilon\}$ such that

$$
\begin{aligned}
\int_{-1}^{1}|x|^{\alpha-\varepsilon} F(\mathrm{~d} x) & =-\int_{-1}^{1}|x|^{-1-\varepsilon} \ln |x| \mathrm{d} x \geqslant \int_{-\varepsilon^{\prime}}^{\varepsilon^{\prime}}|x|^{-1-\varepsilon} \mathrm{d} x=\infty \\
\int_{-1}^{1}|x|^{\alpha+\varepsilon} F(\mathrm{~d} x) & =-\int_{-1}^{1}|x|^{-1+\varepsilon} \ln |x| \mathrm{d} x \leqslant \int_{-1}^{1}|x|^{-1+\varepsilon-\varepsilon^{\prime}} \mathrm{d} x<\infty,
\end{aligned}
$$

i.e., $\alpha$ is the Blumenthal-Getoor index of $L$. Moreover,

$$
\begin{aligned}
A(u) & =\int_{-1}^{1}(\cos (u x)-1)|x|^{-1-\alpha} \ln |x| \mathrm{d} x \\
& \geqslant|u|^{\alpha} \ln (|u|) \int_{-|u|}^{|u|}(1-\cos y)|y|^{-1-\alpha} \mathrm{d} y
\end{aligned}
$$

hence continuity condition (3.1) is not satisfied with index $\alpha$.

Proposition 4.1. Let $L$ be a real-valued Lévy process with characteristic triplet $(b, 0, F)$. Let $0<\beta<2$. 
(i) If $\int_{[-1,1]}|x|^{\beta} F(\mathrm{~d} x)<\infty$ and there exists a constant $C \geqslant 0$ such that $|\operatorname{Im}(A(u))| \leqslant C\left(1+|u|^{\beta}\right)$ then continuity condition (3.1) is satisfied with index $\alpha:=\beta$.

(ii) If $\liminf _{r \downarrow 0} r^{\alpha-2} \int_{[-r, r]}|x|^{2} F(\mathrm{~d} x)>0$, then Gårding condition (3.2) is satisfied with index $\alpha:=\beta$.

P r o o f. Proof of (i). Without loss of generality we may assume $F$ to be symmetric and with support in $[-1,1]$. Inequality (4.3) shows for given $\alpha$ the existence of a positive constant $C(\alpha)$ such that

$$
0 \leqslant \operatorname{Re}(A(u))=\int_{-1}^{1}(1-\cos (u x)) F(\mathrm{~d} x) \leqslant C(\alpha)|u|^{\beta} \int_{-1}^{1}|x|^{\beta} F(\mathrm{~d} x)
$$

which shows assertion (i).

Proof of (ii): From the assumption we get that there exist $N>0$ and a constant $c_{1}>0$ such that

$$
\int_{|v||y| \leqslant \pi} v^{2} y^{2} F(\mathrm{~d} y) \geqslant c_{1}|v|^{\alpha} \quad \text { for all } v \text { with }|v|>N .
$$

As in the proof of [21, Proposition 28.3] we have

$$
\begin{aligned}
\int(1-\cos (v y)) F(\mathrm{~d} y) & =2 \int_{|v||y| \leqslant \pi} \sin ^{2} \frac{v y}{2} F(\mathrm{~d} y)+2 \int_{|v||y|>\pi} \sin ^{2} \frac{v y}{2} F(\mathrm{~d} y) \\
& \geqslant 2 \int_{|v||y| \leqslant \pi} \frac{2}{\pi^{2}} v^{2} y^{2} F(\mathrm{~d} y)+2 \int_{|v||y|>\pi} \sin ^{2} \frac{v y}{2} F(\mathrm{~d} y) \\
& \geqslant c_{2} \int_{|v||y| \leqslant \pi} v^{2} y^{2} F(\mathrm{~d} y) \\
& \geqslant c_{3}|v|^{\alpha} \mathbb{I}_{\{|v|>N\}}
\end{aligned}
$$

with positive constants $c_{2}$ and $c_{3}$.

E x a m ple 4.2. (i) Every pure jump Lévy process which has a Sobolev index has infinite jump activity, i.e., $F(\mathbb{R})=\infty$, which follows from equality (4.1).

(ii) For every Lévy process with Sobolev index $\alpha>1$, its Lévy measure $F$ satisfies $\int_{[-1,1]}|x| F(\mathrm{~d} x)=\infty$. This is an immediate consequence of equality (4.1).

(iii) For every Lévy process with Sobolev index $\alpha>1$, its paths are of bounded variation due to [21, Theorem 21.9] (see also Definition 11.9), which shows that if $\int_{-1}^{1}|x| F(\mathrm{~d} x)<\infty$, then $\mathbf{P}$-a.e. path of $L$ is of bounded variation on $(0, t]$ for every $t>0$.

(iv) Compound Poisson processes do not have a Sobolev index, since their jump activity is finite.

(v) Variance gamma (VG) processes do not have a Sobolev index. Their paths are of bounded variation and their Blumenthal-Getoor index equals 0 . Hence their jump activity is too small to allow for a Sobolev index. 


\subsection{Examples of Lévy processes with Sobolev index.}

$\mathrm{R}$ e $\mathrm{m}$ a r $\mathrm{k}$ 4.1. For $i=1,2$ let $L^{i}$ be two independent Lévy processes with symbol $A^{i}$ and Sobolev index $\alpha_{i}$. Then the sum $L:=L^{1}+L^{2}$ is a Lévy process with symbol $A:=A^{1}+A^{2}$, and obviously the process has a Sobolev index that equals $\max \left\{\alpha_{1}, \alpha_{2}\right\}$.

Ex a m p l e 4.3 (multivariate Lévy processes with Brownian part). $\mathbb{R}^{d}$-valued Lévy processes $L$ with characteristics $(b, \sigma, F)$ with a positive definite matrix $\sigma$ have Sobolev index $\alpha=2$.

P r o o f. Note that

$$
\operatorname{Re}(A(\xi))=\frac{1}{2}\langle\xi, \sigma \xi\rangle+\int(1-\cos \langle\xi, h(y)\rangle) F(\mathrm{~d} y) \geqslant \frac{1}{2}\langle\xi, \sigma \xi\rangle .
$$

Since the matrix $\sigma$ is symmetric and positive definite, we have $\underline{\sigma}|\xi|^{2} \leqslant\langle\xi, \sigma \xi\rangle$ for all $\xi \in \mathbb{R}^{d}$, where $\underline{\sigma}>0$ is the smallest eigenvalue of the matrix $\sigma$. As a consequence, $\underline{\sigma}|\xi|^{2} \leqslant \operatorname{Re}(A(\xi))$ holds, i.e., the Gårding condition (3.2) is satisfied. Continuity condition (3.1) follows immediately from inequality (1.5).

E x a m p l e 4.4 (multivariate Normal Inverse Gaussian (NIG) processes). Let $L$ be an $\mathbb{R}^{d}$-valued NIG process, i.e.,

$$
L_{1}=\left(L_{1}^{1}, \ldots, L_{1}^{d}\right) \sim \mathrm{NIG}_{d}(\alpha, \beta, \delta, \mu, \Delta),
$$

with parameters $\alpha, \delta \geqslant 0, \beta, \mu \in \mathbb{R}^{d}$, and a symmetric positive definite matrix $\Delta \in \mathbb{R}^{d \times d}$ with $\alpha^{2}>\langle\beta, \Delta \beta\rangle$. In [2] multivariate NIG distributions are derived as a subclass of multivariate Generalized Hyperbolic ( $\mathrm{GH}$ ) distributions via a mean variance mixture, more precisely the density of the distribution is defined as

$$
f^{\mathrm{GH}}(x):=\int_{(0, \infty)} f^{\mathrm{N}}(x ; \mu+\beta y, y \Delta) f^{\text {gig }}\left(y ; \lambda, \delta, \sqrt{\alpha^{2}-\langle\beta, \Delta \beta\rangle}\right) \mathrm{d} y,
$$

where $f^{\mathrm{N}}(\cdot, m, \Sigma)$ is the density of the $d$-dimensional normal distribution with mean $m \in \mathbb{R}^{d}$ and covariance matrix $\Sigma$ and

$$
f^{\text {gig }}(\cdot ; \lambda, \delta, \gamma)=\left(\frac{\gamma}{\delta}\right)^{\lambda} \frac{1}{2 K_{\lambda}(\delta \gamma)} x^{\lambda-1} \mathrm{e}^{-\left(\delta^{2} y^{-1}+\gamma^{2} x\right) / 2} \mathbb{I}_{(0, \infty)}(y)
$$

is the density of the generalized inverse Gaussian distribution with parameters $\lambda \in \mathbb{R}$ and $\delta, \gamma>0$ and $K_{\lambda}$ denotes the Bessel function of third kind with parameter $\lambda$. The multivariate normal inverse Gaussian distribution is the GH distribution for $\lambda:=-1 / 2$. The symbol of $L$ is given by

$$
A(u)=i\langle u, \mu\rangle-\delta\left(\sqrt{\alpha^{2}-\langle\beta, \Delta \beta\rangle}-\sqrt{\alpha^{2}-\langle\beta+i u, \Delta(\beta-i u)\rangle}\right),
$$

where by $\langle\cdot, \cdot\rangle$ we denote the product $\left\langle z, z^{\prime}\right\rangle=\sum_{j=1}^{d} z_{j} z_{j}^{\prime}$ for $z \in \mathbb{C}^{d}$, compare, e.g., [11, equation (2.3)]. We verify that $\mathbb{R}^{d}$-valued NIG processes have Sobolev index 1. 
P r o o f. By setting

$$
\begin{aligned}
z & :=\alpha^{2}-\langle\beta-i u, \Delta(\beta-i u)\rangle \\
& =\alpha^{2}-\langle\beta, \Delta \beta\rangle+\langle u, \Delta u\rangle+i\langle\beta, \Delta u\rangle+i\langle u, \Delta \beta\rangle \\
\sqrt{z} & :=\sqrt{\frac{1}{2}(|z|+\operatorname{Re}(z))}+i \frac{\operatorname{Im}(z)}{|\operatorname{Im}(z)|} \sqrt{\frac{1}{2}(|z|-\operatorname{Re}(z))}
\end{aligned}
$$

it follows $|z| \geqslant \alpha^{2}-\langle\beta, \Delta \beta\rangle+\langle u, \Delta u\rangle>0$ and

$$
\begin{aligned}
\operatorname{Re}(A(u)) & =-\delta \sqrt{\alpha^{2}-\langle\beta, \Delta \beta\rangle}+\delta \operatorname{Re}(\sqrt{z}) \\
& =\frac{\delta}{\sqrt{2}} \sqrt{|z|+\operatorname{Re}(z)}-\delta \sqrt{\alpha^{2}-\langle\beta, \Delta \beta\rangle} \\
& \geqslant \delta \sqrt{\lambda_{\min }}|u|-\delta \sqrt{\alpha^{2}-\langle\beta, \Delta \beta\rangle},
\end{aligned}
$$

where $\lambda_{\min }$ denotes the smallest eigenvalue of the matrix $\Delta$. Analogously it follows that $|\operatorname{Re}(u)| \leqslant C_{1}(1+|u|)$ and $|\operatorname{Im}(u)| \leqslant C_{2}(1+|u|)$ with positive constants $C_{1}, C_{2}$, which yields $|A(u)| \leqslant C(1+|u|)$ with a positive constant $C$.

$\mathrm{Ex}$ a m p l e 4.5 (multivariate Cauchy processes). Cauchy processes have Sobolev index 1. This can be seen directly from its symbol,

$$
A(u)=c|u|+i\langle\gamma, u\rangle
$$

which is given, e.g., in [21, Example 2.12].

E x a m p l e 4.6 (generalized Student $t$-processes). Let $L$ be a Lévy process such that the distribution of $L_{1}$ is Student- $t$ with parameters $\mu \in \mathbb{R}$, $\nu>0$, density

$$
\frac{\Gamma((\nu+1) / 2)}{\sqrt{\pi \delta^{2}} \Gamma(\nu / 2)}\left(1+\frac{x-\mu}{\delta^{2}}\right)^{-(\nu+1) / 2}
$$

with $\delta:=f / 4$ and symbol

$$
A(u)=-c-\ln \left\{K_{-\delta}(2 \sqrt{\delta}|u|)\right\}-\ln \left\{|u|^{2 \delta}\right\}+i \mu u .
$$

This generalization of the Student $t$-distribution is defined in [7].

We show that $L$ has Sobolev index 1 .

P r o o f. Since the mapping $u \mapsto A(u)$ is continuous, it is enough to verify the continuity and Gårding inequality for a function that is asymptotically equivalent to $A$. To this aim we insert the asymptotic expansion of the Bessel function

$$
K_{-\delta}(z) \sim \sqrt{\frac{\pi}{2 z}} \mathrm{e}^{-z}\left(1+O\left(|z|^{-1}\right)\right) \quad \text { for }|\arg z|<\frac{3}{2} \pi \text { and }|z| \rightarrow \infty
$$

with the usual notation $f(x) \sim g(x)$ for $|x| \rightarrow \infty$ if $f(x) / g(x) \rightarrow 1$ as $|x| \rightarrow \infty$, see [1, equation (9.7.2)]. The required inequality then follows from the standard analysis. The assertion of Example 4.6 is proved. 
The symbol of a generic real-valued strictly $\alpha$-stable Lévy process is of the form $A(u)=c|u|^{\alpha}$ with a constant $c>0$, see [21, Theorem 14.9]. In this case the Lévy process obviously has Sobolev index $\alpha$. More generally, we have the following example.

According to [21, Definition 24.18] we call an $\mathbb{R}^{d}$-valued stochastic process degenerate if there exists a linear subspace $V \subset \mathbb{R}^{d}$ of smaller dimension $\operatorname{dim} V<d$ containing for every $t>0$ the support of the distribution of $L_{t}$, i.e., for every $x \in \mathbb{R}^{d}$, for which every open subset $U \subset \mathbb{R}^{d}$ containing $x$ satisfies $\mathbf{P}\left(L_{t} \in U\right)>0$, we have $x \in V$. The Lévy process $L$ is called nondegenerate if it is not degenerate.

E x a m p l e 4.7 (multivariate $\alpha$-semi-stable Lévy process). Let $L$ be a nondegenerate $\mathbb{R}^{d}$-valued $\alpha$-semi-stable Lévy process. Then

(a) If $0<\alpha<1$, then $L$ has Sobolev index $\alpha$ if and only if $L$ is strictly $\alpha$-semi-stable;

(b) if $1<\alpha \leqslant 2$, then $L$ has Sobolev index $\alpha$.

Let $L \neq 0$ be a real-valued $\alpha$-stable Lévy process with $\alpha=1$. Then:

(c) If $L$ is strictly 1 -stable and has Sobolev index 1 , then its symbol is of the form

$$
A(u)=c|u|+i \tau u
$$

with $c>0$ and $\tau \in \mathbb{R}$;

(d) $L$ has Sobolev index 1 if and only if $L$ is strictly 1 -stable.

$\mathrm{P}$ r o o f. From [21, Proposition 24.20], we obtain that every nondegenerate $\alpha$-semi-stable Lévy process satisfies the Gårding condition with index $\alpha$.

Let $L$ be an $\alpha$-semi-stable Lévy process with characteristic triplet $(b, \Sigma, F)$, with $\sigma \neq 0$ or $F \not \equiv 0$. From [21, Definition 13.16 and Theorems $13.15,14.1]$ we obtain that $0<\alpha \leqslant 2$. Moreover, we have $\alpha=2$ if and only if $\sigma \neq 0$ and $F \equiv 0$.

For $0<\alpha<2$ with $\alpha \neq 1$, Sato shows in [21, Proposition 14.9] that the symbol $A(\cdot)=-\log \widehat{\mu}(-\cdot)$ is of the form

$$
A(u)=|u|^{\alpha}\left(\eta(u)+i \gamma_{\alpha}(u)\right)+i\left\langle c_{\alpha}, u\right\rangle
$$

with $c_{\alpha} \in \mathbb{R}^{d}, u \mapsto \eta(u)$ nonnegative, continuous on $\mathbb{R}^{d} \backslash\{0\}$ and $\eta(b u)=\eta(u)$ for all $u \in \mathbb{R}^{d}$, and $\gamma_{\alpha}$ real-valued, continuous on $\mathbb{R}^{d} \backslash\{0\}$ with $\gamma_{\alpha}(b u)=\gamma_{\alpha}(u)$ for all $u \in \mathbb{R}^{d}$ with $b=a^{1 / \alpha}>1$. Basic arguments show that the mappings $u \mapsto \eta(u)$ and $u \mapsto \gamma_{\alpha}(u)$ are bounded. We therefore have $\operatorname{Re}(A(u))=$ $|u|^{\alpha} \eta(u)$, where $\eta$ is bounded, and hence $|\operatorname{Re}(A(u))| \leqslant C|u|^{\alpha}$. It remains to derive an adequate upper bound of the imaginary part.

For $0<\alpha<2, \alpha \neq 1$, we have

$$
\operatorname{Im}(A(u))=|u|^{\alpha} \gamma_{\alpha}(u)+\left\langle c_{\alpha}, u\right\rangle
$$

with the bounded function $\gamma_{\alpha}$. 
For $0<\alpha<1$, this shows that $|\operatorname{Im}(A(u))| \leqslant C\left(1+|u|^{\alpha}\right)$ if and only if $c_{\alpha}=0$. According to $[21$, Theorem 14.7 (i)] the latter is the case if and only if the distribution, respectively the Lévy process, is strictly $\alpha$-semi-stable.

For $1<\alpha<2$, due to

$$
\left|\left\langle c_{\alpha}, u\right\rangle\right| \leqslant\left|c_{\alpha}\right||u| \leqslant\left|c_{\alpha}\right|\left(1+|u|^{\alpha}\right),
$$

we obtain $|\operatorname{Im}(A(u))| \leqslant C\left(1+|u|^{\alpha}\right)$ without further restrictions.

Assertions (c) and (d) for $\alpha=1$ are a direct consequence of [21, Theorem 14.15, equation (14.25)], that states that the symbol of a real-valued nonconstant 1-stable Lévy process is of the form

$$
A(u)=c|u|\left(1-i \beta \frac{2}{\pi} \frac{u}{|u|} \log |u|\right)+i \tau u
$$

with $c>0, \beta \in[-1,1]$, and $\tau \in \mathbb{R}$. From this representation of the symbol we can read that $L$ is strictly 1 -stable if and only if $\beta=0$. The representation given in assertion (c) follows as well.

4.3. Tail condition and Sobolev index. It turns out that, if the Lévy measure satisfies a certain tail condition with parameter $1<\alpha<2$, then this index is the Sobolev index of the process.

Lemma 4.1. Let $L$ be a real-valued Lévy process with characteristic triplet $(b, \sigma, F)$ and symbol $A$. Assume $\sigma=0$ and for

$$
\bar{F}(x):=\int_{|y| \geqslant x} F(\mathrm{~d} y)
$$

we assume

$$
\bar{F}(x)=x^{-\alpha} \ell(x)
$$

for some index $0<\alpha<2$ and some function $\ell$ that is bounded on $[-1,1]$ away from zero, i.e., $0<\inf _{-1 \leqslant x \leqslant 1} \ell(x) \leqslant \sup _{-1 \leqslant x \leqslant 1} \ell(x)<\infty$.

Then, the Gairding condition (3.2) is satisfied with index $\alpha$. Moreover, let $1<\alpha<2$, then $\alpha$ is the Sobolev index of the process $L$.

$\mathrm{P}$ r o o f. Notice that by continuity of $u \mapsto A(u)$ around zero, it is enough to assume $|u| \geqslant 1$ in the sequel. Moreover, without loss of generality we assume $F$ has support in $[-1,1]$. We first examine the real part of the symbol by partial integration for the Stieltjes integral,

$$
\begin{aligned}
\operatorname{Re}(A(u)) \geqslant & \int_{-1 /|u|}^{1 /|u|}(1-\cos (|u| x)) F(\mathrm{~d} x) \\
= & \int_{0}^{1 /|u|}|u| \sin (|u| x) \bar{F}(x) \mathrm{d} x-\bar{F}\left(\frac{1}{|u|}\right)(\cos 1-1) \\
& +\lim _{x \rightarrow 0} \bar{F}(x)(\cos (|u| x)-1) \\
\geqslant & |u|^{\alpha} \underline{\ell} \int_{0}^{1} \frac{\sin x}{x^{\alpha}} \mathrm{d} x=C|u|^{\alpha}
\end{aligned}
$$


with a positive constant $C$ and $0<\underline{\ell}:=\inf _{-1 \leqslant x \leqslant 1} \ell(x)$ by nonnegativity of $x \mapsto(1-\cos x)$ and since

$$
\lim _{x \rightarrow 0} \bar{F}(x)(\cos (|u| x)-1)=\lim _{x \rightarrow 0} \frac{\cos (|u| x)-1}{x^{\alpha}} \ell(x)=0 .
$$

In order to examine the absolute value of the real part for $\alpha>1$ of the symbol, we decompose the integral in two parts,

$$
\begin{aligned}
\operatorname{Re}(A(u))= & \int_{-1 /|u|}^{1 /|u|}(1-\cos (|u| x)) F(\mathrm{~d} x) \\
& +\int_{(-1,1) \backslash(-1 /|u|, 1 /|u|)}(1-\cos (|u| x)) F(\mathrm{~d} x) .
\end{aligned}
$$

The boundedness of the first term in absolute value by a constant times $|u|^{\alpha}$ is obvious from the calculations above. For the second term, we get by partial integration

$$
\begin{aligned}
0 & \leqslant \int_{(-1,1) \backslash(-1 /|u|, 1 /|u|)}(1-\cos (|u| x)) F(\mathrm{~d} x) \\
& =\int_{1 /|u|}^{1}|u| \sin (|u| x) \bar{F}(x) \mathrm{d} x+\bar{F}(1)(1-\cos |u|)-\bar{F}\left(\frac{1}{|u|}\right)(1-\cos 1) \\
& \leqslant|u|^{\alpha}\left|\int_{1}^{|u|} y^{-\alpha}(\sin y) \ell\left(\frac{y}{|u|}\right) \mathrm{d} y\right|+2 \bar{F}(1)
\end{aligned}
$$

and

$$
|u|^{\alpha}\left|\int_{1}^{|u|} y^{-\alpha}(\sin y) \ell\left(\frac{y}{|u|}\right) \mathrm{d} y\right| \leqslant \bar{\ell}|u|^{\alpha} \int_{1}^{|u|} y^{-\alpha} \mathrm{d} y \leqslant|u| \frac{\bar{\ell}}{1-\alpha}
$$

with $0<\bar{\ell}:=\sup _{-1 \leqslant x \leqslant 1} \ell(x)$. We now turn to the imaginary part for the case $1 \leqslant \alpha<2$. We integrate by parts,

$$
\begin{aligned}
& \left|\int_{-1}^{1}[u h(x)-\sin (u x)] F(\mathrm{~d} x)\right| \\
& \quad=|| u\left|\int_{-1}^{1}(1-\cos (|u| x)) \bar{F}(x) \mathrm{d} x+\bar{F}(1)(|u|-\sin |u|)\right| \\
& \quad \leqslant|u|^{\alpha} \bar{\ell} \int_{0}^{|u|} \frac{1-\cos x}{x^{\alpha}} \mathrm{d} x+C^{1}(1+|u|)
\end{aligned}
$$

with some constant $C^{1}>0$. Moreover, $0 \leqslant \int_{0}^{1} x^{\alpha}(1-\cos x) \mathrm{d} x<\infty$ for any $\alpha<2$ and there is a constant $C^{2}>0$, independent of $|u|$ such that

$$
0 \leqslant \int_{1}^{|u|} \frac{1-\cos x}{x^{\alpha}} \mathrm{d} x \leqslant \int_{1}^{\infty} \frac{2}{x^{\alpha}} \mathrm{d} x \leqslant C^{2}
$$


for $\alpha>1$. Notice that this is not true for $\alpha=1$. Altogether, there is a constant $C>0$ such that for all $u \in \mathbb{R}$ we have

$$
\left|\int_{-\infty}^{\infty}[u h(x)-\sin (u x)] F(\mathrm{~d} x)\right| \leqslant C\left(1+|u|^{\alpha}\right)
$$

which shows continuity condition (3.1) for $\alpha>1$. Lemma 4.1 is proved.

By further specifying the behavior of $\bar{F}$ around zero, one can derive a condition ensuring the boundedness of the real part of the symbol by $C\left(1+|u|^{\alpha}\right)$ for some positive constant $C$. We refer to [3, Lemma 7.2], where this is derived under the assumption that there are constants $\beta_{0}>0, \beta_{1} \in \mathbb{R}$, and $0<\gamma<\alpha<2$ such that

$$
\bar{F}(x)=x^{-\alpha}\left(\beta_{0}+\beta_{1} x^{\gamma}(1+O(x))\right) .
$$

Tail assumptions on $\bar{F}$ only refer to the symmetric part of the Lévy measure and hence are assumptions on the real part of the symbol. Thus, in order to provide conditions on the Lévy measure that imply the existence of a Sobolev index $0<\alpha \leqslant 1$, one needs to impose additional conditions on the asymmetric part of the Lévy measure.

\subsection{Sobolev index for Lévy processes with absolutely contin-} uous Lévy measure. In this subsection, we study the Sobolev index for real-valued Lévy processes without Brownian part whose Lévy measure has a Lebesgue density. This is, for example, the case for real-valued selfdecomposable Lévy processes.

E x a m p l e 4.8 (selfdecomposable Lévy processes). Let $L$ be a realvalued Lévy process. According to [21, Theorem 15.10], $L$ is selfdecomposable if and only if its Lévy measure $F$ has a Lebesgue density

$$
F(\mathrm{~d} x)=\frac{k(x)}{|x|} \mathrm{d} x
$$

with $k>0$ and $k$ increasing on $(-\infty, 0)$ and decreasing on $(0, \infty)$.

For each $0<\alpha<2$ we provide sufficient conditions on the symmetric and the antisymmetric parts of the density which imply that $\alpha$ is the Sobolev index of the process. More precisely, we assume $F(\mathrm{~d} x)=f(x) \mathrm{d} x$ and set

$$
f_{\mathrm{s}}(x):=\frac{1}{2}(f(x)+f(-x)) \geqslant 0,
$$

the symmetric part of the density. The triangle inequality yields for its antisymmetric part

$$
f_{\mathrm{as}}(x):=f(x)-f_{\mathrm{s}}(x)
$$

that

$$
\left|f_{\text {as }}(x)\right| \leqslant f_{\mathrm{s}}(x)
$$


In $[12$, Lemma 10.4.2] the following case is treated:

$$
f(x) \leqslant C_{+} \frac{1}{|x|^{1+\alpha}} \quad \text { and } \quad f_{\mathrm{s}}(x) \geqslant C_{-} \frac{1}{|x|^{1+\alpha}} \quad \text { for all } 0<|x|<1 .
$$

It turns out that if $\alpha \in(1,2)$, then $\alpha$ is the Sobolev index of $L$. For $\alpha \in(0,1)$, $\alpha$ is the Sobolev index, if we additionally assume $b=\int h(x) F(\mathrm{~d} x)$.

The following example shows that condition (4.13) is not sufficient to ensure the existence of a Sobolev index for the case $\alpha=1$.

Ex a m p l e 4.9. Define the pure jump Lévy process specifying its Lévy measure by $F(\mathrm{~d} x):=x^{-2} \mathbb{I}_{(0,1)}(x) \mathrm{d} x$ with respect to the truncation function $h(x):=x$. Assumption (4.13) is obviously satisfied for $\alpha:=1$. The imaginary part of the related symbol is given by

$$
\begin{aligned}
\operatorname{Im}(A(\xi)) & =\int_{0}^{1}(\sin (\xi x)-\xi x) x^{-2} \mathrm{~d} x=|\xi| \int_{0}^{|\xi|}(\sin x-x) x^{-2} \mathrm{~d} x \\
& \leqslant|\xi|\left(\int_{0}^{\varepsilon}(\sin x-x) x^{-2} \mathrm{~d} x+\int_{\varepsilon}^{|\xi|} x^{-2} \mathrm{~d} x-\int_{\varepsilon}^{|\xi|} x^{-1} \mathrm{~d} x\right) \\
& \leqslant-|\xi|\left(\log |\xi|-\frac{1}{3 \varepsilon^{3}}\right) .
\end{aligned}
$$

We deduce that the absolute value of the imaginary part of the symbol is not bounded linearly in $|\xi|$. The related process hence cannot have a Sobolev index smaller than or equal to 1 .

The real part of the related symbol is given by

$$
\begin{aligned}
\operatorname{Re}(A(\xi)) & =\frac{1}{2} \int_{-1}^{1} \frac{1-\cos (\xi x)}{x^{2}} \mathrm{~d} x \\
& =|\xi| \int_{0}^{|\xi|} \frac{1-\cos (\xi x)}{x^{2}} \mathrm{~d} x \geqslant|\xi| C_{\varepsilon}, \quad \text { for }|\xi|>\varepsilon
\end{aligned}
$$

with $C_{\varepsilon}:=\int_{0}^{\varepsilon} x^{-2}(1-\cos x) \mathrm{d} x>0$. Hence, the real part of the symbol is bounded by a linear function in $|\xi|$, hence this process does not have a Sobolev index.

In the following proposition we derive the Sobolev index for Lévy processes without Brownian part from the behavior of the symmetric and the antisymmetric parts of the Lévy density $f$ around the origin. In particular, the case $f_{\mathrm{s}}=C x^{-2} \mathbb{I}_{[-1,1]}(x)$ is covered.

Proposition 4.2. Let $L$ be a real-valued Lévy process and a special semimartingale with characteristic triplet $(b, 0, F)$ with respect to the truncation function $h(x)=x$. Assume

$$
f_{\mathrm{s}}(x)=\frac{C}{|x|^{1+Y}}+g(x) \quad \text { with } g(x)=O\left(|x|^{-1-Y+\delta}\right) \text { for } x \rightarrow 0
$$


with $\delta>0$. Then, in the following cases, the Lévy process L has Sobolev index $Y$ :

(a) $1<Y<2$;

(b) assume

$$
f_{\text {as }}(x)=O\left(|x|^{-1-\alpha}\right) \quad \text { for } x \rightarrow 0
$$

1) with $\alpha<Y=1$, or

2) let $0<\alpha \leqslant Y<1, \int|x| f(x) \mathrm{d} x<\infty$, and, moreover, $b=\int x F(\mathrm{~d} x)$.

$\mathrm{P}$ r o o f. We treat real and imaginary parts of the symbol separately and start by deriving the upper bound for the real part. Without loss of generality we assume that $f_{\mathrm{s}}$ has support in $[-\varepsilon, \varepsilon]$ and $|u|>1 / \varepsilon$. Assuming $f_{\mathrm{s}}(x)=O\left(1 /|x|^{1+Y}\right)$ for $x \rightarrow 0$, we may further assume without loss of generality that $f_{\mathrm{s}}(x) \leqslant C_{1}|x|^{-1-Y}$ with a constant $C_{1}>0$. We obtain

$$
\begin{aligned}
\operatorname{Re}(A(u)) & =\int_{-\infty}^{\infty}(1-\cos (u x)) f_{\mathrm{s}}(x) \mathrm{d} x \leqslant C_{1} \int_{-\varepsilon}^{\varepsilon} \frac{1-\cos (u x)}{|x|^{1+Y}} \mathrm{~d} x \\
& \leqslant 2 C_{1}|u|^{Y} \int_{0}^{\infty} \frac{1-\cos (u x)}{|x|^{1+Y}} \mathrm{~d} x .
\end{aligned}
$$

The finiteness of the integral is obvious, hence there exists a constant $C \geqslant 0$ with

$$
0 \leqslant \operatorname{Re}(A(u))=A^{f_{\mathrm{s}}}(u) \leqslant C\left(1+|u|^{Y}\right) \quad \text { for all } u \in \mathbb{R} .
$$

If we assume instead $f_{\mathrm{s}}(x)=C /|x|^{1+Y}+g(x)$ with $g(x)=O\left(1 /|x|^{1+Y-\delta}\right)$ for $x \rightarrow 0$ with some $\delta>0$ and $C>0$, then there exist constants $C_{1}>0$, $C_{2} \geqslant 0$, and $Y^{\prime} \in(0, Y)$ such that

$$
\operatorname{Re}(A(u))=A^{f_{\mathrm{s}}}(u) \geqslant C_{1}|u|^{Y}-C_{2}\left(1+|u|^{Y^{\prime}}\right) \quad \text { for all } u \in \mathbb{R},
$$

which follows directly from inequality (4.15) applied to $g$ and from

$$
\operatorname{Re}(A(u)) \geqslant C_{3}(\varepsilon) \int_{-\varepsilon}^{\varepsilon} \frac{1-\cos (u x)}{|x|^{1+Y}} \mathrm{~d} x-C_{4}\left(1+|u|^{Y-\delta}\right)
$$

together with

$$
\int_{-\varepsilon}^{\varepsilon} \frac{1-\cos (u x)}{|x|^{1+Y}} \mathrm{~d} x \geqslant 2|u|^{Y} \int_{0}^{1} \frac{1-\cos x}{|x|^{1+Y}} \mathrm{~d} x .
$$

In each of the three cases in the proposition, the Gårding condition (3.2) follows directly from inequality (4.16).

We now examine the absolute value of the imaginary part of the symbol,

$$
|\operatorname{Im}(A(u))|=\left|\int(\sin (u x)-u x) f_{\mathrm{as}}(x) \mathrm{d} x+b u\right|,
$$


for which we derive upper bounds. Inequality (4.11) allows us to conclude

$$
\begin{aligned}
& \int_{(-\varepsilon, \varepsilon)^{c}}|u x-\sin (u x)|\left|f_{\text {as }}(x)\right| \mathrm{d} x \\
& \quad \leqslant|u| \int_{(-\varepsilon, \varepsilon)^{c}}|x|\left|f_{\text {as }}(x)\right| \mathrm{d} x+\int_{(-\varepsilon, \varepsilon)^{c}}\left|f_{\text {as }}(x)\right| \mathrm{d} x \\
& \quad=: C_{1}(\varepsilon)|u|+C_{2}(\varepsilon)
\end{aligned}
$$

with nonnegative constants $C_{1}(\varepsilon)$ and $C_{2}(\varepsilon)$. From the assumption on $f_{\text {as }}$ we get for $\varepsilon>0$ small enough,

$$
\begin{gathered}
\int_{-\varepsilon}^{\varepsilon}|u x-\sin (u x)|\left|f_{a s}(x)\right| \mathrm{d} x \leqslant C(\varepsilon) \int_{-\varepsilon}^{\varepsilon}|u x-\sin (u x)| \frac{1}{|x|^{1+\alpha}} \mathrm{d} x \\
=C(\varepsilon)|u|^{\alpha} \int_{-\varepsilon|u|}^{\varepsilon|u|} \frac{|x-\sin x|}{|x|^{1+\alpha}} \mathrm{d} x \\
=2 C(\varepsilon)|u|^{\alpha}\left(\int_{0}^{1} \frac{x-\sin x}{|x|^{1+\alpha}} \mathrm{d} x+\int_{1}^{\varepsilon|u|} \frac{x-\sin x}{|x|^{1+\alpha}} \mathrm{d} x\right),
\end{gathered}
$$

where the first integral is finite, since $\alpha<2$. For $\alpha \neq 1$ we have

$$
\int_{1}^{\varepsilon|u|} \frac{x-\sin x}{|x|^{1+\alpha}} \mathrm{d} x \leqslant \int_{1}^{\varepsilon|u|}\left(x^{-\alpha}+x^{-1-\alpha}\right) \mathrm{d} x \leqslant C_{3}(\varepsilon)\left(1+|u|^{-\alpha}\right)
$$

with some constant $C_{3}(\varepsilon)>0$. Combining these estimates and fixing some $\varepsilon>0$, we obtain the assertion: If $f_{\text {as }}(x)=O\left(1 /|x|^{1+\alpha}\right)$ for $x \rightarrow 0$ with $0<\alpha$ and $\alpha \neq 1$, then there exist constants $C, C_{1} \geqslant 0$ such that for every $u \in \mathbb{R}$,

$$
|\operatorname{Im}(A(u))| \leqslant C\left(1+|u|+|u|^{\alpha}\right) \leqslant C_{1}\left(1+|u|^{\max \{1, \alpha\}}\right) .
$$

Inequality (4.17) shows the continuity condition (3.1) for $Y \in[1,2)$ if $f_{\text {as }}$ is of the form (4.14).

Assume $Y \in(1,2)$. It is a direct consequence of equality (4.11) that assumption (4.14) with $\alpha:=Y$ follows from (4.14) which shows assertion (a) of the proposition.

For the case $Y=1$, condition (4.14) for $\alpha<1$ is imposed from which part 1) of assertion (b) follows.

Let us assume $Y \in(0,1)$. In this case the first-order term is dominant and the continuity condition (3.1) does not follow from inequality (4.17). Since $f_{\text {as }}$ is antisymmetric and

$$
\int\left|x f_{\text {as }}(x)\right| \mathrm{d} x \leqslant \int|x| f(x) \mathrm{d} x<\infty
$$

by equality (4.11) and the assumption, we obtain

$$
\operatorname{Im}(A(u))=\int \sin (u x) f_{\text {as }}(x) \mathrm{d} x-u \int x f(x) \mathrm{d} x+b u .
$$


Furthermore, since the drift is given by $\int x f(x) \mathrm{d} x$, we have

$$
\begin{aligned}
|\operatorname{Im}(A(u))| & =\left|\int \sin (u x) f_{\text {as }}(x) \mathrm{d} x\right| \\
& \leqslant \int_{(-\varepsilon, \varepsilon)^{c}}\left|f_{\text {as }}(x)\right| \mathrm{d} x+\int_{-\varepsilon}^{\varepsilon}|\sin (u x)|\left|f_{\text {as }}(x)\right| \mathrm{d} x .
\end{aligned}
$$

Hence by the assumption on $f_{\text {as }}$ we obtain for $0<\alpha \leqslant Y$ that there exist positive constants $C(\varepsilon), C_{1}(\varepsilon)$ such that

$$
\begin{aligned}
|\operatorname{Im}(A(u))| & \leqslant \int_{(-\varepsilon, \varepsilon)^{c}}\left|f_{\text {as }}(x)\right| \mathrm{d} x+\int_{-\varepsilon}^{\varepsilon}|\sin (u x)|\left|f_{\text {as }}(x)\right| \mathrm{d} x \\
& \leqslant F\left((-\varepsilon, \varepsilon)^{c}\right)+C(\varepsilon) \int_{-\varepsilon}^{\varepsilon} \frac{|\sin (u x)|}{|x|^{1+\alpha}} \mathrm{d} x \\
& \leqslant C_{1}(\varepsilon)+C(\varepsilon)|u|^{\alpha} \int_{-\infty}^{\infty} \frac{|\sin x|}{|x|^{1+\alpha}} \mathrm{d} x .
\end{aligned}
$$

Finiteness of $\int_{-\infty}^{\infty}|\sin (x)| /|x|^{1+\alpha} \mathrm{d} x$ yields continuity condition (3.1) and thus part 2) of assertion (b).

E x a m p l e 4.10 (univariate GH processes). Real-valued Generalized Hyperbolic (GH) processes have Sobolev index 1.

P r o o f. $L$ has Lévy density

$$
f^{\mathrm{GH}}(x)=C_{1} \frac{1}{x^{2}}+C_{2} \frac{1}{|x|}+C_{3} \frac{1}{x}+\frac{o(|x|)}{x^{2}}, \quad x \downarrow 0,
$$

see [19, Proposition 2.18]. Hence the symmetric part $f_{\mathrm{s}}^{\mathrm{GH}}$ and the antisymmetric part $f_{\mathrm{as}}^{\mathrm{GH}}$ of $f^{\mathrm{GH}}$ are of the form

$$
f_{\mathrm{s}}^{\mathrm{GH}}(x)=\frac{C}{|x|^{2}}+O\left(|x|^{-1}\right) \quad \text { and } \quad f_{\mathrm{as}}^{\mathrm{GH}}(x)=O\left(|x|^{-1}\right) \quad \text { for } x \rightarrow 0 .
$$

The assertion follows from part (b) of Proposition 4.2.

E x a m p l e 4.11 (univariate generalized tempered stable Lévy process). A generalized tempered stable Lévy process $L$ with parameters $C_{-}$, $C_{+} \geqslant 0$ such that $C_{-}+C_{+}>0$ and $G, M>0$ and $Y_{-}, Y_{+}<2$, is a Lévy process that has no Brownian part and its Lévy measure $F^{\text {temp }}$ is given by its Lebesgue density

$$
f^{\text {temp }}(x)= \begin{cases}\frac{C_{-}}{|x|^{1+Y_{-}}} \mathrm{e}^{G x} & \text { for } x<0, \\ \frac{C_{+}}{|x|^{1+Y_{+}}} \mathrm{e}^{-M x} & \text { for } x \geqslant 0,\end{cases}
$$

compare [18]. For $C_{ \pm}=0$ we set $Y_{ \pm}:=0$ and call $(b, 0, F)$ its characteristic triplet with respect to the truncation function $h$. 
(i) If $\alpha:=\max \left\{Y_{+}, Y_{-}\right\}>1$, then $L$ has Sobolev index $\alpha$.

(ii) If $Y:=Y_{-}=Y_{+}=1$ and $C_{-}=C_{+}$, then $L$ is a CGMY process with Sobolev index $Y=1$.

(iii) If $0<\alpha:=\max \left\{Y_{+}, Y_{-}\right\}<1$, then $L$ has Sobolev index $\alpha$ if $b:=C_{+} M^{1-Y_{+}} \Gamma\left(Y_{+}\right)-C_{-} G^{1-Y_{-}} \Gamma\left(Y_{-}\right)$for the truncation function $h(x)=x$.

In the special case $C:=C_{+}=C_{-}$and $Y:=Y_{+}=Y_{-}$, where $L$ is a CGMY process with parameters $C, G, M$, and $Y$. We get the following assertions.

(iv) If $Y \in[1,2)$, then $L$ has Sobolev index $Y$.

(v) If $Y \in(0,1)$ and $b=C \Gamma(-Y)\left(M^{Y-1}-G^{Y-1}\right)$ with respect to the truncation function $h(x)=x$, then $L$ has Sobolev index $Y$.

In another special case, for $C_{-}=0,0<Y_{+}<1$, and $b=$ $\int_{0}^{\infty} x f^{\text {temp }}(x) \mathrm{d} x, L$ is a subordinator and from case (iii) we deduce that $L$ has Sobolev index $Y$.

P r o o f. For the symmetric part $f_{\mathrm{s}}^{\text {temp }}$ of the density we have by its definition (4.10)

$$
f_{\mathrm{s}}^{\mathrm{temp}}(x)=\frac{C_{-} \mathrm{e}^{-G|x|}}{2|x|^{1+Y_{-}}}+\frac{C_{-} \mathrm{e}^{-M|x|}}{2|x|^{1+Y_{+}}}=\frac{C}{|x|^{1-\max \left\{Y_{-}, Y_{+}\right\}}}+O\left(|x|^{-1-\alpha+\varepsilon}\right)
$$

for some $0<\varepsilon<\alpha$ and where the positive constant $C$ is given by $C:=C_{-} / 2$ if $Y_{-}>Y_{+}, C:=C_{+} / 2$ if $Y_{+}>Y_{-}$, and $C:=C_{+}$for $Y_{+}=Y_{-}$.

For the antisymmetric part $f_{\text {as }}^{\text {temp }}$ of the density, we obtain from its definition (4.11)

$$
f_{\mathrm{as}}^{\mathrm{temp}}(x)=\frac{\operatorname{sign} x}{2}\left(C_{+} \frac{\mathrm{e}^{-M|x|}}{|x|^{1+Y_{+}}}-C_{-} \frac{\mathrm{e}^{-G|x|}}{|x|^{1+Y_{-}}}\right)=O\left(|x|^{-1-\max \left\{Y_{-}, Y_{+}\right\}}\right) .
$$

From equations (4.18) and (4.19), Proposition 4.2 yields assertion (i) and assertion (iii) for

$$
\begin{aligned}
b=\int x f^{\text {temp }}(x) \mathrm{d} x & =-C_{-} \int_{-\infty}^{0}|x|^{Y_{-}} \mathrm{e}^{-G|x|} \mathrm{d} x+C_{+} \int_{0}^{\infty}|x|^{Y_{+}} \mathrm{e}^{-M|x|} \mathrm{d} x \\
& =C_{+} M^{1-Y_{+}} \Gamma\left(Y_{+}\right)-C_{-} G^{1-Y_{-}} \Gamma\left(Y_{-}\right) .
\end{aligned}
$$

For the case $\max \left\{Y_{-}, Y_{+}\right\}=1$, equation (4.19) is not sufficient. In view of counterexample 4.9 we need that the singularity around zero of the antisymmetric part is of a smaller order. This is only possible for $Y_{+}=Y_{-}=: Y$ and $C_{-}=C_{+}=: C$. In this case, the tempered stable process is indeed a CGMY process with parameters $C, G, M$, and $Y=1$, and we obtain

$$
\left|f_{\text {as }}^{\text {CGMY }}(x)\right|=\frac{C}{2} \frac{\left|\mathrm{e}^{-G|x|}-\mathrm{e}^{-M|x|}\right|}{|x|^{1+Y}}=O\left(|x|^{-Y}\right) \quad \text { for }|x| \rightarrow 0 .
$$

From Proposition 4.2 (b) we obtain that $L$ has Sobolev index $Y=1$, i.e., assertion (ii). 
The special case of a CGMY process, assertions (iv) and (v), are a direct consequence of assertions (i)-(iii). It is implied by [21, Theorem 10.1] that the other specification defines subordinator. The assertions of Example 4.11 are proved.

In Example 4.11 (iii) and (v), the drift is chosen as $b=\int h(x) \mathrm{d} x$ in order to guarantee a Sobolev index in the case $\alpha<1$. If the drift is chosen differently, the first-order term of the imaginary part is dominating and the process does not have a Sobolev index.

$\mathrm{R}$ e $\mathrm{mark}$ 4.2. In mathematical finance, when the stock price is modeled as an exponential of a Lévy process, $S_{t}=S_{0} \mathrm{e}^{L_{t}}$ with a Lévy process $L$ such that $\mathbf{E} S_{t}<\infty$ and deterministic continuously compounding interest rate $r$, the drift has to be chosen as $b:=r-\sigma / 2-\int_{-\infty}^{\infty}\left(\mathrm{e}^{x}-1-h(x)\right) F(\mathrm{~d} x)$, where the characteristic triplet is given by $(b, \sigma, F)$ with respect to the truncation function $h$.

This choice of the drift is essential, since it guarantees that the model is arbitrage-free and that the discounted fair prices are (local) martingales. The related Kolmogorov backward equations are then used to determine option prices. They can only be parabolic, if the underlying process has a Sobolev index bigger than or equal to 1.

In particular, when $L$ is a CGMY process, Example 4.11 and Corollary 3.2 show that the related Kolmogorov backward equations are parabolic with respect to $\left(\widetilde{H}^{\alpha / 2}(D), L^{2}(D),\left(\widetilde{H}^{\alpha / 2}(D)\right)^{*}\right)$, if $\alpha:=Y \geqslant 1$.

5. Numerical illustration. As a numerical example we calculate the solution of a boundary value problem and demonstrate the effect of the Sobolev index on the performance of numerical procedures to solve the Kolmogorov backward equation. To this aim, we chose $\left(L_{t}\right)_{t \geqslant 0}$ as a CGMY process and vary the parameter $Y$, see Example 4.11 for the definition of a CGMY-process. Denote by $\mathscr{G}$ CGMY its infinitesimal generator and set $\mathscr{A}^{\mathrm{CGMY}}:=-\mathscr{G}^{\mathrm{CGMY}}$.

Example 4.11 shows that $\alpha:=Y$ is the Sobolev index of $L$ in the case $Y \geqslant 1$. Hence, Corollary 3.2 yields the existence of a unique variational solution $u \in W^{1}\left(0, T ; \widetilde{H}^{\alpha / 2}(-N, B) ; L^{2}(-N, B)\right)$ of the parabolic boundary value problem

$$
\partial_{t} u+\mathscr{A}^{\mathrm{CGMY}} u=0, \quad u(0)=\mathbb{1}_{(-N, B)} .
$$

We compute the solution $u$ numerically for various different values of $Y$. More precisely the parameters are $C=0.0156, G=0.0767, M=7.55$, $B=6.9, N=13, T=0.5$, and $Y=0.1, \ldots, 1.9$. As numerical procedure, we use a wavelet-Galerkin scheme programmed in MATLAB. In space, we discretized the solution with a wavelet-Galerkin scheme, the time-discretization is based on an $h p$-discontinuous method. In order to study convergence, we 
compute the discrete solutions $u^{l}$ for different levels $l$, which determine the grid size: in discretization level $l$, one has $1+2^{l+1}$ grid points in space.

We compute the error $\varepsilon^{l}:=\left\|u^{L}(T, \cdot)-u^{l}(T, \cdot)\right\|_{L^{2}}$ in the norm of $L^{2}((-N, B))$ of the numerical solutions $u^{l}$ for the different levels $l$ with respect to the finest solution $u^{L}$ for the highest level $L=14$.

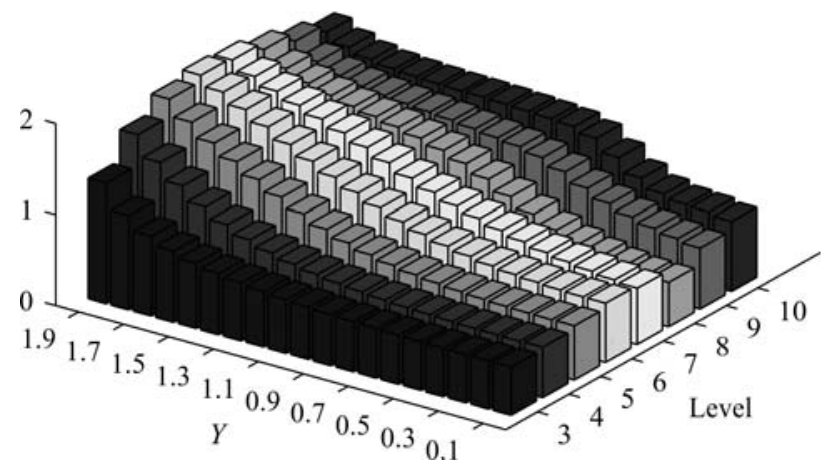

Fig. 5.1. Values of $\operatorname{EOC}(l)$ for different values of $Y$ and different levels $l$ with fixed level $L=14$.

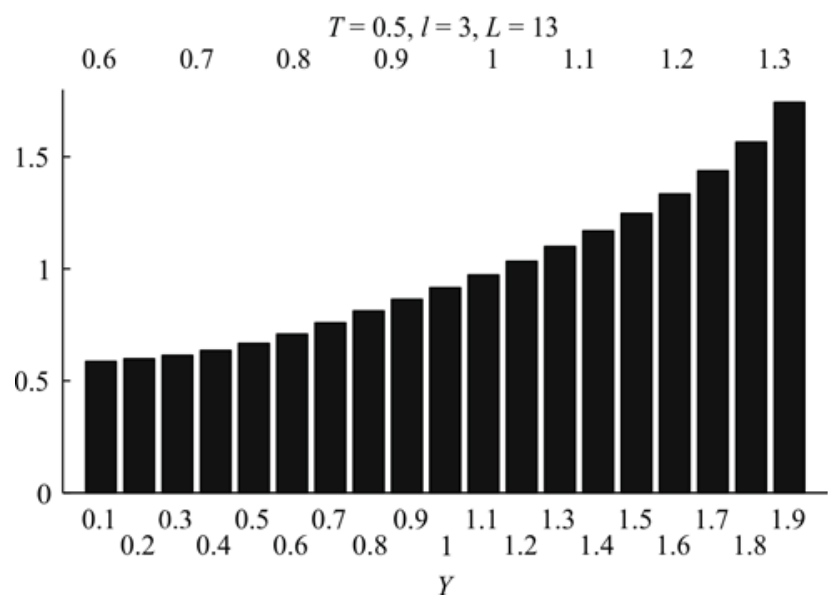

Fig. 5.2. Average (1/9) $\sum_{l=3}^{l=11} \operatorname{EOC}(l)$ for different values of $Y$.

Figures 5.1 and 5.2 display the Experimental Order of Convergence (EOC)

$$
\operatorname{EOC}(l):=\log _{2} \frac{\varepsilon_{\mathrm{rel}}^{l, L}}{\varepsilon_{\mathrm{rel}}^{l+1, L}}, \quad \text { where } \varepsilon_{\mathrm{rel}}^{l, L}:=\frac{\left\|u^{L}(T, \cdot)-u^{l}(T, \cdot)\right\|_{L^{2}}}{\left\|u^{L}(T, \cdot)\right\|_{L^{2}}}
$$

with respect to the $L^{2}$-norm for different values of $Y$.

Both figures indicate a dependence of the order of convergence on the Sobolev index $Y$. The figures indicate that the convergence is faster for higher values of $Y$, as it was expected from the fact that the solution $u$ is more regular for higher values of the Sobolev index. 


\section{REFERENCES}

1. Абрамович М., Стиган И. Справочник по специальным функциям с формулами, графиками и математическими таблицами. М.: Наука, 1979, 830 с.

2. Barndorff-Nielsen O.E. Exponentially decreasing distributions for the logarithm of particle size. - Proc. Roy. Soc. Lond. A, 1977, v. 353, № 1674, p. 401-419.

3. Belomestny D., Panov V. Estimation of the activity of jumps in time-changed Lévy models. - Electron. J. Statist., 2013, v. 7, p. 2970-3003.

4. Бенсусан А., Лионс Ж.-Л. Импульсное управление и квазивариационные неравенства. М.: Наука, 1987, 597 с.

5. Böttcher B., Schilling R., Wang J. Lévy-type processes: construction, approximation and sample path properties. - Lecture Notes in Math., 2013, v. 2099, p. 1-199.

6. Eberlein E., Glau K. Variational solutions of the pricing PIDEs for European options in Lévy models. - Appl. Math. Finance, 2014, v. 21, № 5, p. 417-450.

7. Eberlein E., von Hammerstein E.A. Generalized hyperbolic and inverse Gaussian distributions: limiting cases and approximation of processes. - Seminar on Stochastic Analysis, Random Fields and Applications IV. Ed. by R. C. Dalang, M. Dozzi, and F. Russo. Basel: Birkhäuser, 2004, p. 221-264. (Progr. Probab., v. 58.)

8. Эскин Г. И. Краевые задач для эллиптических псевдодифференциальных уравнений. М.: Наука, 1973, 230 с.

9. Glau K. Feynman-Kac-Darstellung zur Optionspreisbewertung in Lévy-Modellen. $\mathrm{PhD}$ thesis, Universität Freiburg, 2010.

10. Glau K. Feynman-Kac formula for Lévy processes with discontionuous killing rate. arXiv:1502.07531

11. Hammerstein E.-A. Generalized Hyperbolic Distributions: Theory and Applications to CDO Pricing. PhD thesis, Universität Freiburg, 2010.

12. Hilber N., Reichmann N., Schwab C., Winter C. Computational Methods for Quantitative Finance. Finite Element Methods for Derivative Pricing. Berlin: Springer, 2013, 299 p.

13. Jacob N. Pseudo-Differential Operators and Markov Processes. V. II: Generators and their Potential Theory. London: Imperial College Press, 2002, 453 p.

14. Matache A.-M., Nitsche P.-A., Schwab C., Wavelet Galerkin pricing of American options on Lévy driven assets. - Quantitative Finance, 2005, v. 5, № 4, p. 403-424.

15. Matache A.-M., Schwab C., Wihler T.P. Fast numerical solution of parabolic integrodifferential equations with applications in finance. - SIAM J. Sci. Comput., 2005, v. 27 , № 2 , p. 369-393.

16. Matache A.-M., von Petersdorff T., Schwab C. Fast deterministic pricing of options on Lévy driven assets. — Math. Modell. Numer. Anal., 2004, v. 38, № 1, p. 37-71.

17. Picard J. On the existence of smooth densities for jump processes. - Probab. Theory Related Fields, 1996, v. 105, № 4, p. 481-511.

18. Poirot J., Tankov P. Monte Carlo option pricing for tempered stable (CGMY) processes. - Asia-Pacific Financial Markets, 2006, v. 13, № 4, p. 327-344.

19. Raible S. Lévy Processes in Finance: Theory, Numerics, and Empirical Facts. PhD thesis, Universität Freiburg, 2000.

20. Rudin W. Real and Complex Analysis. New York: McGraw-Hill, 1966, 412 p.

21. Sato K.-I. Lévy Processes and Infinitely Divisible Distributions. Cambridge: Cambridge Univ. Press, 1999, 486 p.

22. Thomée V. Galerkin Finite Element Methods for Parabolic Problems. Berlin: Springer, 2006, $370 \mathrm{p}$.

23. von Petersdorff T., Schwab C. Wavelet discretizations of parabolic integrodifferential equations. - SIAM J. Numer. Anal., 2003, v. 41, № 1, p. 159-180.

24. Wloka J. Partial Differential Equations. Cambridge: Cambridge Univ. Press, 1987, $518 \mathrm{p}$

25. Zeidler E. Nonlinear Functional Analysis and its Applications. V. II/A: Linear Monotone Operators. New York: Springer-Verlag, 1990, 467 p.

Поступила в редакцию 19.VI.2013

Исправленный вариант 27.IV.2014 